\title{
Interest Rate Rules and Macroeconomic Stability under Heterogeneous Expectations
}

\author{
Mikhail Anufrieva Tiziana Assenzab,a \\ Cars Hommes $^{\mathrm{a}}$ Domenico Massaro ${ }^{\mathrm{a}}$ \\ a CeNDEF, School of Economics, University of Amsterdam, \\ Roetersstraat 11, NL-1018 WB Amsterdam, Netherlands \\ b ITEMQ, Catholic University of Milan, \\ Via Necchi 5, 20123 Milan, Italy
}

M.Anufriev@uva.nl; P. +31 20525 4248; F. +31 205254349 tiziana.assenza@unicatt.it; P. +39 027234 2483; F. +39 0272342923

C.H.Hommes@uva.nl; P. +31 20525 4246; F. +31 205254349

D.Massaro@uva.nl; F. +31 205254349

This Draft: May 2011 
Interest Rate Rules and Heterogeneous Expectations

Corresponding author: Cars Hommes

CeNDEF, School of Economics, University of Amsterdam,

Roetersstraat 11, NL-1018 WB Amsterdam, Netherlands.

C.H.Hommes@uva.nl; P. +31 20525 4246; F. +31 205254349 


\begin{abstract}
The recent macroeconomic literature stresses the importance of managing heterogeneous expectations in the formulation of monetary policy. We use a simple frictionless DSGE model to investigate inflation dynamics under alternative interest rate rules when agents have heterogeneous expectations and update their beliefs based on past performance as in Brock and Hommes (1997). The stabilizing effect of different monetary policies depends on the ecology of forecasting rules (i.e., the composition of the set of predictors), on agents' sensitivity to differences in forecasting performance and on how aggressively the monetary authority sets the nominal interest rate in response to inflation. In particular, if the monetary authority only responds weakly to inflation, a cumulative process with rising inflation is likely. On the other hand, a Taylor interest rate rule that sets the interest rate more than point for point in response to inflation stabilizes inflation dynamics, but does not always lead the system to converge to the rational expectations equilibrium as multiple equilibria may persist.
\end{abstract}

JEL codes: E52, D83, D84, C62.

Keywords: Heterogeneous Expectations, Monetary Policy, Cumulative Process, Taylor Rule.

Acknowledgment: We would like to thank participants of the POLHIA meeting at Università La Sapienza, Rome, May 15, 2008, to the 14th International Conference on Computing in Economics and Finance, Paris, June 26 - 28, 2008 and to a seminar at the University of Technology, Sydney, November 2009, for stimulating discussions. We also wish to thank Buz Brock, Peter Howitt, Florian Wagener and two referees for helpful comments on an earlier draft of this paper. Financial support from the EU $7^{\text {th }}$ framework collaborative project "Monetary, Fiscal and Structural Policies with Heterogeneous Agents (POLHIA)", grant no. 225408, is gratefully acknowledged. None of the above are responsible for errors in this paper. 


\section{Introduction}

The rational representative agent approach is still the core assumption in macroeconomics. In contrast, in behavioral finance models with bounded rationality and heterogeneous expectations have been developed as a concrete alternative to the standard rational representative agent approach. These heterogeneous agents models mimic important observed stylized facts in asset returns, such as fat tails, clustered volatility and long memory, as discussed, e.g., in the extensive surveys of LeBaron (2006) and Hommes (2006). Although bounded rationality and adaptive learning have become increasingly important in macroeconomics, most models still assume a representative agent who is learning about the economy (see, e.g., Evans and Honkapohja (2001) and Sargent (1999) for extensive overviews) and thus ignore the possibility of heterogeneity in expectations and its consequences for monetary policy and macroeconomic stability. Some recent examples of macro models with heterogeneous expectations include Brock and de Fontnouvelle (2000), Evans and Honkapohja (2003, 2006), Branch and Evans (2006), Honkapohja and Mitra (2006), Berardi (2007), Tuinstra and Wagener (2007), Brazier, Harrison, King, and Yates (2008), Assenza and Berardi (2009), Branch and McGough (2009, 2010), and Lines and Westerhoff (2010). Carroll (2003), Mankiw, Reis, and Wolfers (2003), Branch (2004) and Pfajfar and Santoro (2010) recently provided empirical evidence in support of heterogeneous expectations using survey data on inflation expectations, while Hommes, Sonnemans, Tuinstra, and van de Velden (2005), Adam (2007), Pfajfar and Zakelj (2010) and Assenza, Heemeijer, Hommes, and Massaro (2011) find evidence for heterogeneity in learning-to-forecast laboratory experiments with human subjects.

The importance of managing expectations for conducting monetary policy has been recognized and stressed, e.g., in Woodford (2003) (p. 15). However, the question how to manage expectations when forecasting rules are heterogeneous has hardly been addressed. The aim of our paper is to investigate whether the Cen- 
tral Bank can enhance macroeconomic stability, in the presence of heterogeneous expectations about future inflation, by implementing simple interest rate rules. In particular, we investigate how the ecology of potential forecasting rules affects the stabilizing properties of a simple Taylor rule. Moreover we study how, in a world where expectations are heterogeneous, the aggressiveness of the monetary authority in responding to fluctuations of the inflation rate affects these stabilizing properties. See also De Grauwe (2010) for a recent discussion on how heterogeneous expectations may affect monetary policy.

In order to study the potential (de-)stabilizing role of heterogeneous expectations we use a simple frictionless model of inflation. In our stylized model agents form expectations about the future rate of inflation using different forecasting rules. We employ the heterogeneous expectations framework of Brock and Hommes (1997), where the ecology of forecasting rules is disciplined by endogenous, evolutionary selection of strategies with agents switching between forecasting rules on the basis of their past performance.

Our paper relates to the literature on interest rate rules and price stability under learning dynamics. Howitt (1992) pointed out that interest rate rules that do not react aggressively to inflation are deceptive to people trying to acquire rational expectations through learning. Indeed, in a world in which any departure of expected inflation from its equilibrium level causes an overreaction of actual inflation and generates a misleading signal for the agents, a forecasting rule that tries to learn from past mistakes will lead the economy away from equilibrium causing a cumulative process of accelerating inflation or deflation. ${ }^{1}$ Howitt (1992) shows that the cumulative process arises for any plausible backward looking learning rule ${ }^{2}$ in a

\footnotetext{
${ }^{1}$ Friedman (1968) argued that interest rate pegging is not a sound monetary policy, even if the chosen interest rate is consistent with the Wicksellian natural rate of interest, and hence consistent with a rational expectation equilibrium. For even in that case, any small discrepancy between inflation expectations of the public and the ones required for the realization of the rational expectations equilibrium will drive expectations even farther from consistency with the rational expectations equilibrium. Howitt (1992) reformulates Friedman's argument as a failure of convergence of learning dynamics to rational expectations equilibrium.

${ }^{2}$ Adaptive learning in the sense of Evans and Honkapohja (2001) belongs to the class of learning rules considered by Howitt (1992).
} 

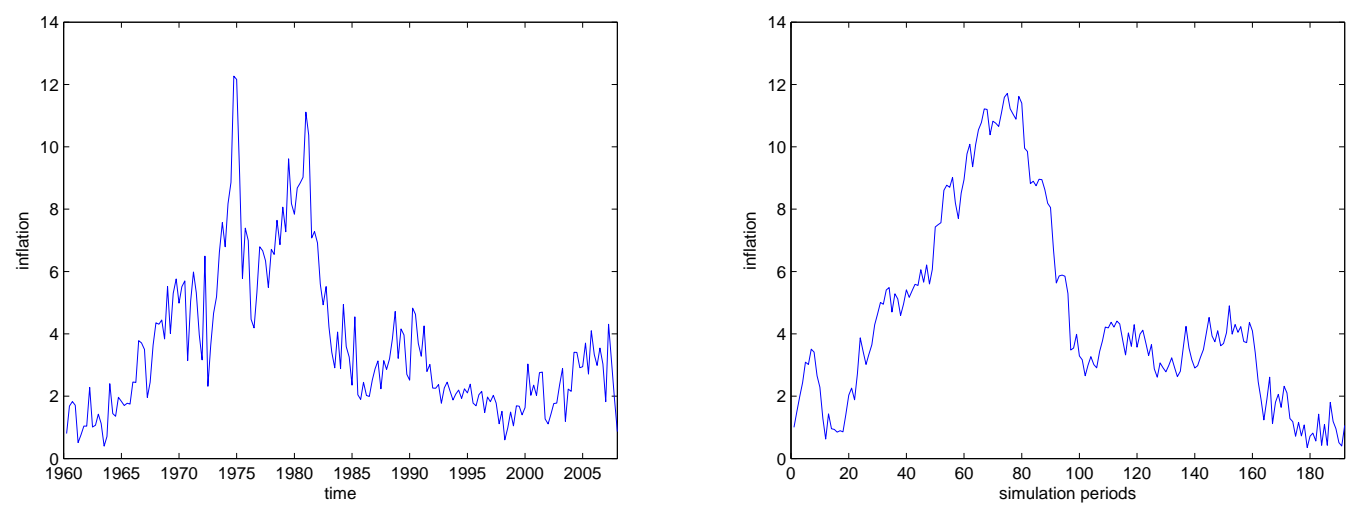

Figure 1: Inflation time series. Left: US annualized inflation, quarterly data for a period 1960-2007. Right: Simulated data.

homogeneous expectations setting. Moreover he shows that, by reacting more than point for point to inflation when setting the interest rate, the monetary authority can avoid the cumulative process. This monetary policy rule has become known as the "Taylor principle", after Taylor (1993).

The present paper investigates the dynamical consequences of committing to an interest rate feedback rule in a world with endogenously evolving heterogeneous expectations. As we will see, the answer whether a Taylor rule can stabilize the cumulative process depends in interesting ways on the ecology of forecasting rules and on how aggressively the monetary authority adjusts the interest rate in response to inflation.

To illustrate the empirical relevance, we performed stochastic simulations of our model in order to reproduce some qualitative features of US inflation time series. In Fig. 1 we confront the simulated dynamics of a stochastic version of our model buffeted with shocks to economic fundamentals (right panel) with actual time series of US inflation (left panel). The model is simulated for 192 periods corresponding to quarters. An immediate observation is that the simulated inflation series is highly persistent. Hence, even in a frictionless DSGE model heterogeneous expectations rules may lead to highly persistent inflation (e.g. Milani (2007)). The monetary policy rule in the simulation exhibits a structural break in period 80 , 
when the Central Bank changes the coefficient (measuring its aggressiveness in responding to actual inflation) of the interest rate rule. This break corresponds to the policy shift instituted by Fed chairman Paul Volcker in 1979. Before the structural break, in setting the interest rate the Central Bank responds relatively weakly to inflation. In our nonlinear model with heterogeneous expectations, when the Central Bank only responds weakly to inflation multiple steady states arise and, as a consequence, self-fulfilling expectations contribute to and reinforce a strong rise in inflation initially triggered by shocks to fundamentals, consistent with US data. In period $T=80$, after the structural break, the Central Bank modifies the monetary policy rule to respond more aggressively, i.e., adapts the nominal interest rate more than point for point in response to inflation. Because of this policy change some of the high level steady states disappear and inflation stabilizes to low levels, consistent with US data. ${ }^{3}$ Our model, thus, explains the strong rise in US inflation between 1960 and 1980 as being triggered by shocks to economic fundamentals (such as the Oil shocks in 1973 and 1979), reinforced by evolutionary selection among heterogeneous forecasting rules under a too weakly responding Taylor rule in the pre-Volcker period, and the subsequent strong decline in US inflation data between 1980 and 2007 (the Great Moderation) enforced by a more aggressive interest rate rule.

The paper is organized as follows. Section 2 briefly recalls the ideas behind the cumulative process and recalls the micro-founded benchmark model. The model with heterogeneous expectations is introduced in Section 3, where we test the validity of the Taylor principle both in the case of a small number of constant forecasting rules (e.g., 3 or 5) and in the case of an arbitrarily large number of rules, applying the notion of Large Type Limit (Brock, Hommes, and Wagener

\footnotetext{
${ }^{3}$ In our model under the Taylor rule inflation does not necessarily converge to the RE level, as different co-existing equilibria may persist. This result differs from the standard representative agent adaptive learning literature, where the interest rate rules that satisfy the Taylor principle lead to a unique, E-stable RE equilibrium, see, e.g., Bullard and Mitra (2002). Preston (2005) shows that, under adaptive learning, monetary policy rules obeying the Taylor principle lead to a unique, E-stable RE equilibrium when agents have heterogeneous but symmetric information sets.
} 
(2005)). In Section 4 we discuss a calibration of our model to US inflation data. Finally, Section 5 concludes.

\section{Interest Rate Rules and Cumulative Process}

In this section we recall the instability problem implied by the Wicksellian cumulative process. We follow Benhabib, Schmitt-Grohé, and Uribe (2002), Woodford (2003), Cochrane $(2005,2010)$ and many others in describing a frictionless economy. Consumers maximize expected present discounted value of utility

$$
\max \widehat{E}_{t} \sum_{j=0}^{\infty} \delta^{j} u\left(C_{t+j}\right)
$$

where $\widehat{E}_{t} C_{t+j}$ indicates subjective expectations of private agents in period $t$ regarding consumption $C_{t+j}$ in period $t+j$, and $0<\delta<1$ is a discount factor. Consumers face a budget constraint given by

$$
P_{t} C_{t}+B_{t}=\left(1+i_{t-1}\right) B_{t-1}+P_{t} Y
$$

where $P_{t}$ is the price of the good, $B_{t}$ represents holdings of one-period bonds, $i_{t}$ is the nominal interest rate and $Y$ is a constant nonstorable endowment. We assume that the government issues no debt so that $B_{t}=0$ and that public expenditure is equal to zero. The market clearing condition thus requires $C_{t}=Y$.

The first order condition for the optimization problem is given by the Euler equation

$$
u_{c}\left(C_{t}\right)=\delta\left(1+i_{t}\right) \widehat{E}_{t} u_{c}\left(C_{t+1}\right)\left(\frac{P_{t}}{P_{t+1}}\right)
$$

together with the budget constraint holding as an equality at each date. ${ }^{4}$ Using

\footnotetext{
${ }^{4}$ As standard, we require that the agent's subjective transversality condition, given by $\lim _{j \rightarrow \infty} \widehat{E}_{t} \delta^{j} u_{c}\left(C_{t+j}\right) \frac{B_{t+j}}{P_{t+j}}=0$,
} 
the consumers' Euler equation and the market clearing condition we have that the interest rate follows a Fisher relation

$$
\frac{1}{1+i_{t}}=\delta \widehat{E}_{t}\left(\frac{P_{t}}{P_{t+1}}\right)
$$

which can be linearized to get

$$
i_{t}=r+\widehat{E}_{t} \pi_{t+1}
$$

where $\pi_{t+1}$ is the inflation rate and $r$ is the constant real interest rate. ${ }^{5}$

Assume that the monetary authority responds to the inflation rate according to the following Taylor rule:

$$
i_{t}=r+\phi_{\pi} \pi_{t}
$$

We can solve the model by substituting out the nominal interest rate, in order to get the equilibrium condition

$$
\pi_{t}=\frac{1}{\phi_{\pi}} \widehat{E}_{t} \pi_{t+1}
$$

Following Howitt (1992) it is possible to show that interest rate rules with a reaction coefficient $\phi_{\pi}<1$ lead to a cumulative process when expectations are revised in an adaptive, boundedly rational way. To illustrate the failure of interest rules reacting less than point for point to inflation, let us assume that the economy is in the zero steady state and people expect a small amount of inflation. Equilibrium condition (2.3) implies that realized inflation will be even higher than expected when $\phi_{\pi}<1$. This means that the signal that agents receive from the market is misleading. Even though inflation was overestimated with respect to the equilibrium level, realized inflation suggests that agents underestimated it. Any reasonable rule that tries is satisfied ex-post. See Evans, Honkapohja, and Mitra (2003) for a discussion.

${ }^{5}$ In equilibrium we have that the real interest rate is constant and given by $r=\delta^{-1}-1$. 
to learn from past mistakes will then lead agents to expect even higher inflation, causing a cumulative process of accelerating inflation. Similarly, if people expect a deflation, an interest rate rule with a reaction coefficient $\phi_{\pi}<1$ will lead to a cumulative process of accelerating deflation.

The actual inflation dynamics depends, of course, on the forecasting rule that agents use to form their expectations. As an illustrative example, consider the case of naive expectations, i.e., when agents expect that past inflation will persist in the future, $\widehat{E}_{t} \pi_{t+1}=\pi_{t-1}$. Using (2.3) we can describe the dynamics under naive expectations by the linear equation

$$
\pi_{t}=\frac{1}{\phi_{\pi}} \pi_{t-1}
$$

whose unique steady state corresponds to the RE equilibrium, $\pi^{*}=0$. This steady state is, however, unstable, and thus any initial non-equilibrium level of inflation will lead to a cumulative process.

When the Central Bank implements a monetary policy rule that makes the nominal interest rate respond to the rate of inflation more than point for point, i.e., it obeys the "Taylor principle", the cumulative process can be avoided. Assume that in the example above the Central Bank adopts a policy rule with a reaction coefficient $\phi_{\pi}>1$. It is immediately clear that for such a Taylor rule the RE equilibrium is globally stable and the cumulative process will not arise.

Finally, notice that under RE the expected inflation coincides with the actual inflation so that dynamics can be described by:

$$
\pi_{t}=\frac{1}{\phi_{\pi}} \pi_{t+1}
$$

It's easy to see that when $\phi_{\pi}<1$, the RE steady state $\pi^{*}=0$ is indeterminate, i.e., it is approached by many RE paths, while when $\phi_{\pi}>1$ the RE equilibrium is determinate. $^{6}$

\footnotetext{
${ }^{6}$ This result is well known also for the full version of the New Keynesian model with frictions,
} 


\section{Interest Rate Feedback Rules with Fundamen- talists and Biased Beliefs}

Will the cumulative process arise in an economy where agents have heterogeneous expectations about the future level of the inflation rate? Will an interest rate rule that obeys the Taylor principle succeed in stabilizing inflation? To address these questions we employ the framework of Adaptive Belief Systems proposed in Brock and Hommes (1997) to model heterogeneous expectations. Assume that agents can form expectations choosing from $H$ different forecasting rules. We denote by $\widehat{E}_{h, t} \pi_{t+1}$ the forecast of inflation by rule $h$. The fraction of agents using forecasting rule $h$ at time $t$ is denoted by $n_{h, t}$. Assuming linear aggregation of individual expectations, ${ }^{7}$ actual inflation in equation (2.3) is given by

$$
\pi_{t}=\frac{1}{\phi_{\pi}} \sum_{h=1}^{H} n_{h, t} \widehat{E}_{h, t} \pi_{t+1} .
$$

The evolutionary part of the model describes the updating of beliefs over time. Fractions are updated according to an evolutionary fitness measure. The fitness measures of all strategies are publicly available, but subject to noise. Fitness is derived from a random utility model and given by

$$
\widetilde{U}_{h, t}=U_{h, t}+\varepsilon_{h, i, t},
$$

where $U_{h, t}$ is the deterministic part of the fitness measure and $\varepsilon_{h, i, t}$ represent IID idiosyncratic noise at date $t$, across types $h=1, \ldots, H$ and agents $i$. Assuming

see Woodford (2003).

${ }^{7}$ Averaging of individual forecasts represents a first-order approximation to general nonlinear aggregation of heterogeneous expectations. Recent papers following the same approach include Adam (2007), Arifovic, Bullard, and Kostyshyna (2007), Brazier, Harrison, King, and Yates (2008), Branch and McGough (2010) and De Grauwe (2010). Aggregate dynamics under heterogeneous expectations can be decoupled from the dynamics of the wealth distribution if one assumes, e.g., that the markets are complete and all assets are in zero net supply. Branch and McGough (2009) developed a New Keynesian model with heterogeneous expectations in which the laws of motion of aggregate variables are comparable to those obtained under homogeneous rational expectations. The model used in this paper can be considered as a frictionless version of their model. 
that the noise $\varepsilon_{h, i, t}$ is drawn from a double exponential distribution, in the limit as the number of agents goes to infinity, the probability that an agent chooses strategy $h$ is given by the well known discrete choice fractions (see Manski and McFadden (1981)):

$$
n_{h, t}=\frac{e^{\beta U_{h, t-1}}}{\sum_{h=1}^{H} e^{\beta U_{h, t-1}}} .
$$

Note that the higher the fitness of a forecasting rule $h$, the higher the probability that an agent will select strategy $h$. The parameter $\beta$ is called the intensity of choice and reflects the sensitivity of the mass of agents to selecting the optimal prediction strategy. The intensity of choice $\beta$ is inversely related to the variance of the noise term. The case $\beta=0$ corresponds to the situation of infinite variance in which differences in fitness can not be observed, so agents do not switch between strategies and all fractions are constant and equal to $1 / H$. The case $\beta=\infty$ corresponds to the situation without noise in which the deterministic part of the fitness can be observed perfectly and in every period all agents choose the best predictor. A natural performance measure is past squared forecast errors

$$
U_{h, t-1}=-\left(\pi_{t-1}-\widehat{E}_{h, t-2} \pi_{t-1}\right)^{2}-C_{h}
$$

where $C_{h}$ is the per period information gathering cost of predictor $h$.

Consider an environment in which agents can choose between different constant "steady state" predictors to forecast future inflation. This represents a situation in which agents roughly know the fundamental steady state of the economy, but they are boundedly rational and disagree about the correct value of the fundamental inflation rate. Forecasting the $\mathrm{RE}$ equilibrium value of inflation, $\pi^{*}=0$, requires some cognitive efforts and information gathering costs, which will be incorporated in the cost $C \geq 0 .{ }^{8}$ Realized inflation and expectations will co-evolve over time

\footnotetext{
${ }^{8}$ In our model formulation the fundamental steady state is deterministic, but the model can be reformulated with a stochastic fundamental. The costs $C \geq 0$ then represent information gathering costs of a time-varying fundamental steady state.
} 
and evolutionary selection based on reinforcement learning will decide which forecasting rule performs better and will survive in the evolutionary environment. The class of constant forecasts is extremely simple, but it should be emphasized that it is also broad because it includes all possible point-predictions of next period's inflation level. Moreover, learning to forecast laboratory experiments with human subjects show that individuals use very simple rules, including constant predictors (see Assenza, Heemeijer, Hommes, and Massaro (2011); Hommes (2011)). For this simple class of rules it will be possible to obtain analytical results under heterogeneous expectations. We will consider simple examples with only a few rules as well as examples with a large number, even a continuum of rules, representing an ecology of predictors including all possible steady state predictions.

\subsection{Evolutionary Dynamics with Few Constant Belief Types}

As a first step we consider the simplest scenario in which agents can choose between three different forecasting rules:

$$
\begin{aligned}
& \widehat{E}_{1, t} \pi_{t+1}=0, \\
& \widehat{E}_{2, t} \pi_{t+1}=b, \\
& \widehat{E}_{3, t} \pi_{t+1}=-b,
\end{aligned}
$$

with bias parameter $b>0$. Type 1 agents believe that the inflation rate will always be at its RE level. Type 2 agents have a positive bias, expecting that inflation will be above its fundamental level, while type 3 agents have a negative bias, expecting an inflation level below the fundamental value. ${ }^{9}$ Assuming that the equilibrium

\footnotetext{
${ }^{9}$ Notice that this example has "symmetric" beliefs, in the sense that the positive and negative biases are exactly balanced around the REE. The main reason why we assume symmetry of the belief types is that under such an assumption the REE is among the steady states of the dynamical system. Thus, with symmetric belief types we can address the important question of stability of the REE. We stress, however, that symmetry of beliefs is not essential for many qualitative features of the model, e.g., bifurcations of multiple steady state with increase of intensity of choice. The insight of the model can therefore be used to study the consequences of policy changes (after which the symmetry would be lost since the belief types would not respond to the policy shift immediately), as we do in Section 4.
} 
predictor is available at cost $C \geq 0$ and substituting the forecasting rules of the three types into (3.1) we get

$$
\pi_{t}=\frac{1}{\phi_{\pi}}\left(n_{2, t} b-n_{3, t} b\right)=f_{\beta}\left(\pi_{t-1}\right)
$$

where fractions are updated according to the discrete choice model (3.2), that is,

$$
n_{2, t}=\frac{e^{-\beta\left(\pi_{t-1}-b\right)^{2}}}{Z_{t-1}}, \quad n_{3, t}=\frac{e^{-\beta\left(\pi_{t-1}+b\right)^{2}}}{Z_{t-1}},
$$

and

$$
Z_{t-1}=e^{-\beta\left(\pi_{t-1}^{2}+C\right)}+e^{-\beta\left(\pi_{t-1}-b\right)^{2}}+e^{-\beta\left(\pi_{t-1}+b\right)^{2}} .
$$

Dynamics in (3.4) is described by a 1-dimensional map. This map $f_{\beta}$ is increasing, bounded and symmetric w.r.t. point $\pi=0$, see Appendix A. It implies that the dynamics always have a steady state $\pi^{*}=0$, which is the RE equilibrium. However, this RE steady state may not be globally or even locally stable. In some cases the dynamics may converge to other stable steady states, which will be denoted as $\pi^{+}>0$ and $\pi^{-}=-\pi^{+}<0$. In what follows we provide a complete analysis of global dynamics of (3.4) and show how these dynamics depend on parameters $b$, $C, \beta$ and $\phi_{\pi}$. We will distinguish between two cases: a "low cost" case, when $C<b^{2}$, which includes the case of a freely available equilibrium predictor, and a "high cost" case, when $C \geq b^{2}$.

Let us start with the case in which the fundamental predictor has small or even zero costs. We introduce two constants $\phi_{\pi}^{w}(b, C)=\phi_{\pi}^{w}<\phi_{\pi}^{a}=\phi_{\pi}^{a}(b, C)$, described in Eqs. (B.4) and (B.5) of Appendix B, respectively. Three different situations can be distinguished on the basis of the "strength" of the policy reaction coefficient $\phi_{\pi}$. When $\phi_{\pi}<\phi_{\pi}^{w}$, we define the monetary policy implemented by the Central Bank as weak. The corresponding dynamics are described in Proposition 3.1 below. When $\phi_{\pi}^{s}<\phi_{\pi}<\phi_{\pi}^{a}$, the monetary policy is defined as moderate, whereas when 

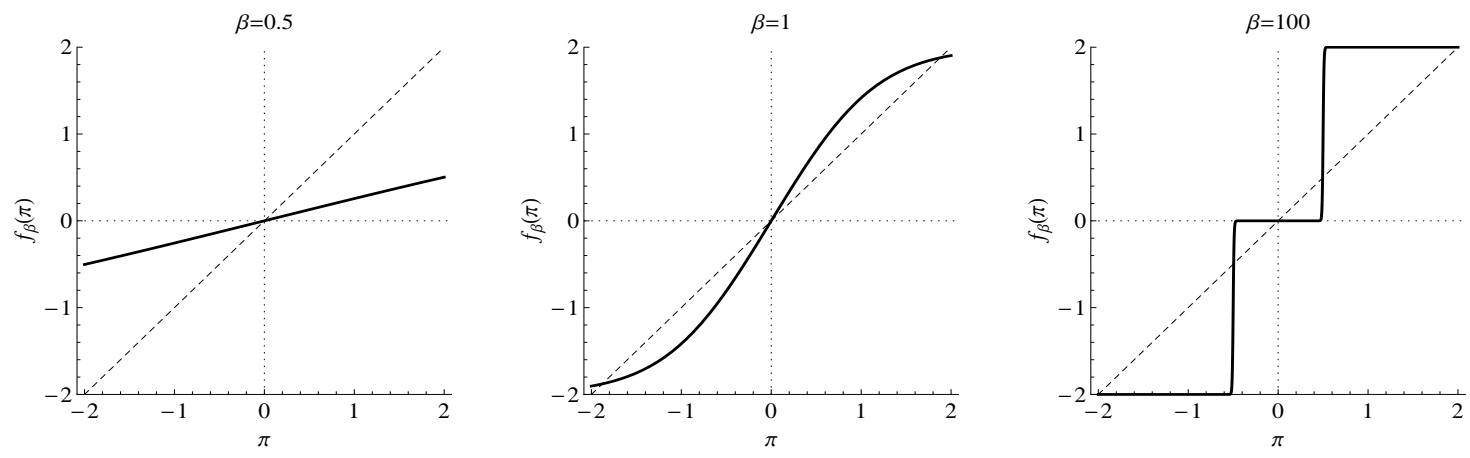

Figure 2: Low information costs case. The map $f_{\beta}$ in the system with 3 belief types for different values of $\beta$. The parameters values are $b=1, C=0$, and $\phi_{\pi}=0.5$.

$\phi_{\pi}^{s}>\phi_{\pi}^{a}$, the monetary policy is defined as aggressive. ${ }^{10}$ These cases are analyzed in Propositions 3.2 and Proposition 3.3, respectively.

Proposition 3.1. Let $C<b^{2}$ ("low costs") and $\phi_{\pi}<\phi_{\pi}^{w}$ ("weak policy"). Then values $0<\beta_{1}^{*} \leq \beta_{2}^{*}<\beta_{3}^{*} \leq \beta_{4}^{*}$ exist such that

- for $\beta<\beta_{1}^{*}$ the RE steady state is unique and globally stable.

- for $\beta_{2}^{*}<\beta<\beta_{3}^{*}$ three steady states exist, the unstable RE steady state $\pi^{*}$, and two other stable non-RE steady states, $\pi^{+}$and $\pi^{-}$.

- for $\beta>\beta_{4}^{*}$ five steady states exist, three steady states are locally stable ( $\pi^{*}$, $\pi^{+}$and $\pi^{-}$) and two other steady states are unstable;

Proof. See Appendix B, where we also provide numerical evidence that $\beta_{3}^{*}=\beta_{4}^{*}$ and that when $\phi_{\pi}$ is small enough, $\beta_{1}^{*}=\beta_{2}^{*}$.

Fig. 2 shows the maps $f_{\beta}$ under a weak monetary policy for small, medium, and high values of the intensity of choice $\beta$. We set $\operatorname{costs} C=0$ and the policy reaction coefficient $\phi_{\pi}=0.5$. When the intensity of choice is relatively low, there exists only one steady state, the RE steady state, which is globally stable. For low intensity of choice agents are more or less evenly distributed over the different forecasting

\footnotetext{
${ }^{10}$ When $C=0$, the threshold values of the monetary policy reaction coefficient $\phi_{\pi}^{w}$ and $\phi_{\pi}^{a}$ are independent of $b$ (see Appendix B). In particular we have that, when $C=0, \phi_{\pi}^{w}=0.93$ and $\phi_{\pi}^{a}=2$.
} 
rules, thus realized inflation will remain relatively close to the fundamental steady state. As the intensity of choice increases, the RE steady state loses stability and two new stable non-fundamental steady states are created. However, as $\beta$ increases further, we have that the RE steady state becomes stable again and two additional unstable steady states are created. In the case of low costs for fundamentalists, we thus have three stable steady states, $\pi^{+}>0, \pi^{-}<0$ and also $\pi^{*}=0$, for high values of the intensity of choice $\beta$. The economic intuition behind the fact that non-fundamental steady states exist for high intensity of choice is simple (cf. Proposition B.2 in Appendix B). Suppose that the intensity of choice is high and that, at time $t$, inflation rate $\pi_{t}$ is close to the optimistic belief, that is, $\pi_{t} \approx b$. The positive bias forecast will perform better than the negative bias and the fundamental belief. Therefore, when the intensity of choice is high, almost all agents will forecast inflation with the positive bias, i.e., $n_{2, t+1} \approx 1$, implying that $\pi_{t+1} \approx b / \phi_{\pi}$. The same intuition explains existence of a negative non-fundamental steady state for high intensity of choice. However, with low costs $C$, when the system is close to the fundamental steady state, a relatively cheap fundamental rule is the best predictor, causing more agents to switch to the fundamental rule and leading the dynamics to converge to the RE equilibrium $\pi^{*}=0$.

When the Central Bank implements a "moderate" interest rate rule, the following applies

Proposition 3.2. Let $C<b^{2}$ ("low costs") and $\phi_{\pi}^{w}<\phi_{\pi}<\phi_{\pi}^{a}$ ("moderate policy"). Then values $0<\beta_{1}^{*} \leq \beta_{2}^{*}$ exist such that

- for $\beta<\beta_{1}^{*}$ the RE steady state is unique and globally stable;

- for $\beta>\beta_{2}^{*}$ five steady states exist, three steady states $\left(\pi^{*}, \pi^{+}\right.$and $\left.\pi^{-}\right)$are locally stable and two other steady states are unstable;

Proof. See Appendix B, where we also provide numerical evidence that $\beta_{1}^{*}=\beta_{2}^{*}$. 

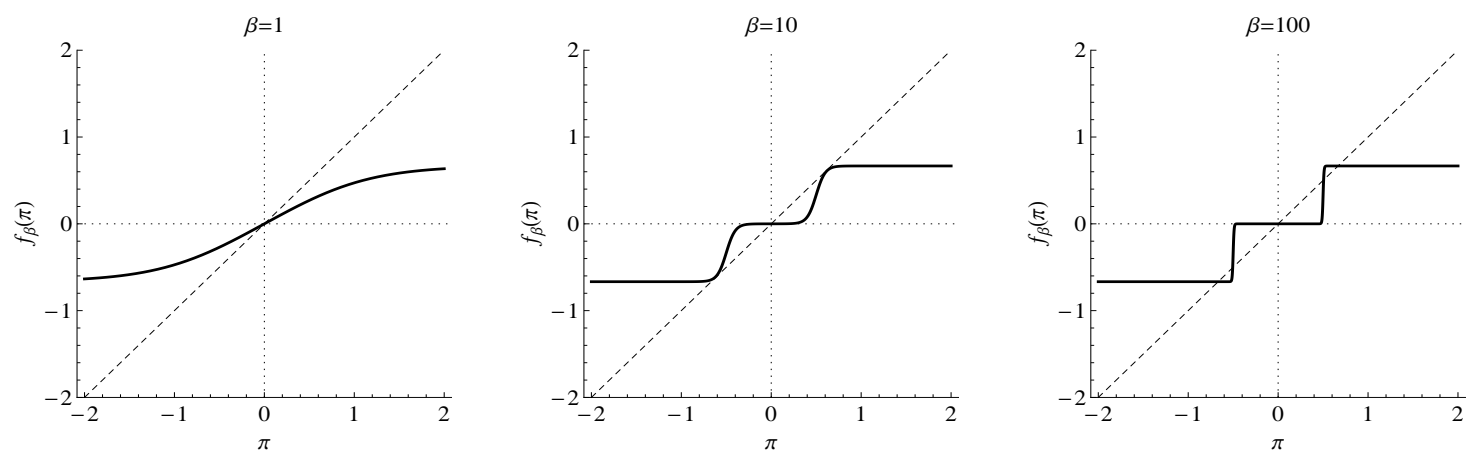

Figure 3: Low information costs case. The map $f_{\beta}$ in the system with 3 belief types for different values of $\beta$. The parameters values are $b=1, C=0$, and $\phi_{\pi}=1.5$.

Fig. 3 shows the maps $f_{\beta}$ under a moderate monetary policy for small, medium, and high values of the intensity of choice $\beta$. We set costs $C=0$ and the policy reaction coefficient $\phi_{\pi}=1.5$. As before, when the intensity of choice $\beta$ is relatively low we have a unique globally stable fundamental steady state $\pi^{*}=0$. When the intensity of choice increases, the RE equilibrium remains locally stable and four additional steady states, two stable and two unstable are created. The difference with the previous case, i.e., when the monetary authority implements a "weak" interest rate rule, is that the zero steady state does not lose local stability. Therefore, a relatively strong reaction of the interest rate to inflation in a neighborhood of the RE steady state, lead the dynamics to converge to the fundamental equilibrium. However, when the intensity of choice is high and inflation is out of the basin of attraction of $\pi^{*}$ and close, for example, to the optimistic belief, the implemented policy is not aggressive enough in reacting to inflation causing more and more agents to adopt the positive bias forecast and leading the economy to converge to the positive non-fundamental steady state. The same intuition explains the existence of a negative non-fundamental steady state.

When the Central Bank implements an "aggressive" monetary policy we have the following

Proposition 3.3. Let $C<b^{2}$ ("low costs") and $\phi_{\pi}>\phi_{\pi}^{a}$ ("aggressive policy"). Then the RE steady state is unique and globally stable for any $\beta$. 

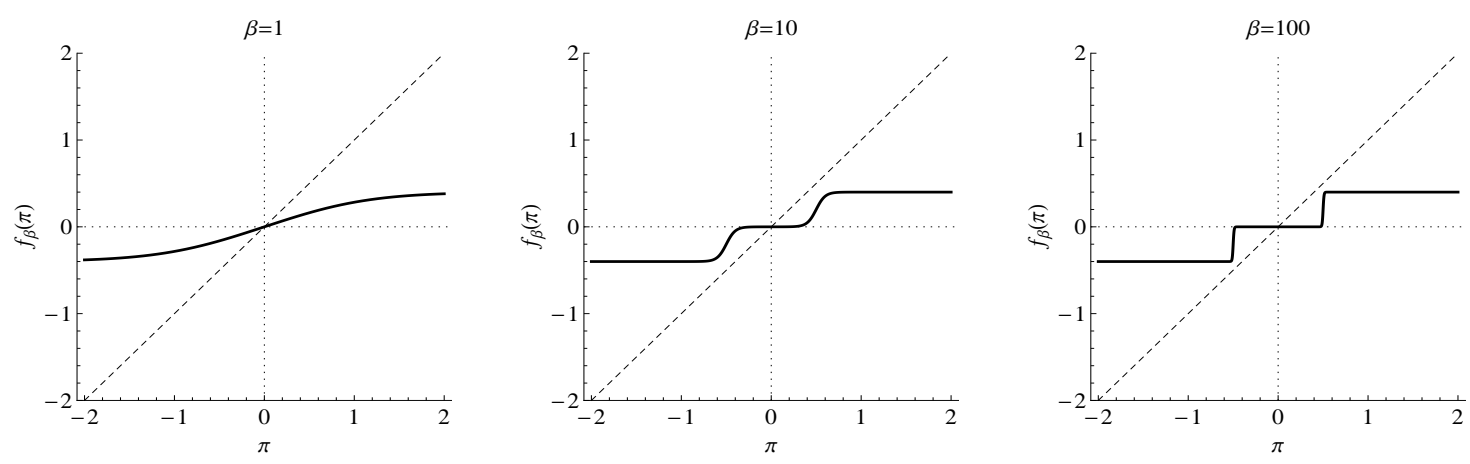

Figure 4: Low information costs case. The map $f_{\beta}$ in the system with 3 belief types for different values of $\beta$. The parameters values are $b=1, C=0$, and $\phi_{\pi}=2.5$.

Proof. See Appendix B.

Fig. 4 shows the maps $f_{\beta}$ under an aggressive monetary policy for small, medium, and high values of the intensity of choice $\beta$. We set costs $C=0$ and the policy reaction coefficient $\phi_{\pi}=2.5$. By reacting aggressively to inflation, the monetary authority manages to avoid multiplicity of equilibria and keeps the RE equilibrium globally stable. The intuition for this result is simple. Consider the limiting case $\beta=\infty$ and suppose that, at time t, inflation rate $\pi_{t}$ is close to the optimistic belief, that is, $\pi_{t} \approx b$. When $\beta=\infty$, all agents will forecast inflation with the positive bias, i.e., $n_{2, t+1}=1$, implying that $\pi_{t+1}=b / \phi_{\pi}$. When $\operatorname{costs} C=0$ the threshold value $\phi_{\pi}^{a}=2$. For $\phi_{\pi}>2$ we have that $\pi_{t+1}<b / 2$, so that the zero predictor will be the closest to realized inflation. This leads all agents to adopt the fundamental forecasting rule, driving thus the system to the RE equilibrium. The result in Proposition 3.3 implies that, given parameters $b$ and $C$, the Central Bank can always implement an interest rate rule that satisfies $\phi_{\pi}>\phi_{\pi}^{a}>1$ and keep the RE equilibrium unique and globally stable.

Consider now the case in which the fundamental predictor has relatively high costs. Then

Proposition 3.4. Let $C \geq b^{2}$ ("high costs"). Then there exists $\beta^{*}$ such that

- for $\beta<\beta^{*}$ the RE steady state is unique and globally stable; 

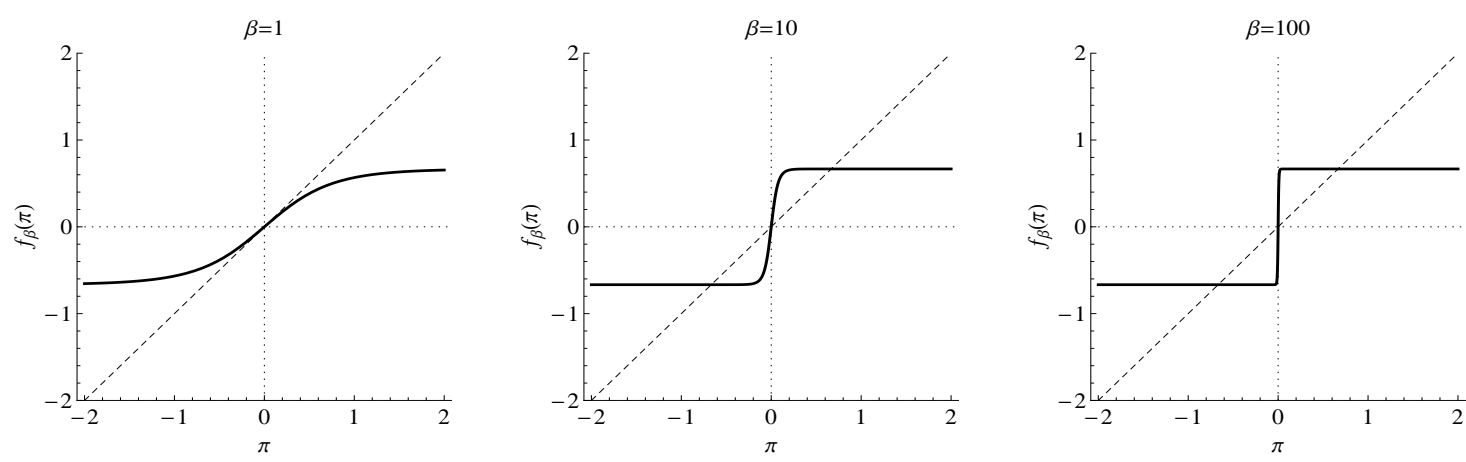

Figure 5: High information costs case. The map $f_{\beta}$ in the system with 3 belief types for different values of $\beta$. The parameters values are $b=1, C=1$, and $\phi_{\pi}=1.5$.

- $\operatorname{for} \beta>\beta^{*}$ three steady state exist, the unstable RE steady state $\pi^{*}$, and two other locally stable non-RE steady states, $\pi^{+}$and $\pi^{-}$.

Proof. See Appendix B.

Fig. 5 shows the maps $f_{\beta}$ when costs $C$ are relatively high for small, medium, and high values of the intensity of choice $\beta$. We set costs $C=1$ and the policy reaction coefficient to $\phi_{\pi}=1.5$. When the intensity of choice is relatively low, there exists only one steady state, the RE steady state, which is globally stable. As before, for low intensity of choice agents are more or less evenly distributed over the different forecasting rules, thus realized inflation will remain relatively close to the fundamental steady state. As the intensity of choice increases, the RE steady state loses stability and two new stable non-fundamental steady state are created. When costs for the fundamental predictor are high, the Central Bank can not implement a monetary policy that keeps the RE equilibrium stable when the intensity of choice is relatively high. In fact, the costs for the fundamental predictor are so high that they overcome the forecasting error of the biased beliefs, even when inflation is close to the RE equilibrium. However, the more aggressive the monetary policy, the higher the bifurcation value $\beta^{*}$ and the smaller the distance between the stable non-fundamental equilibria and the RE steady state.

A similar analysis can be made for other examples with larger number of con- 

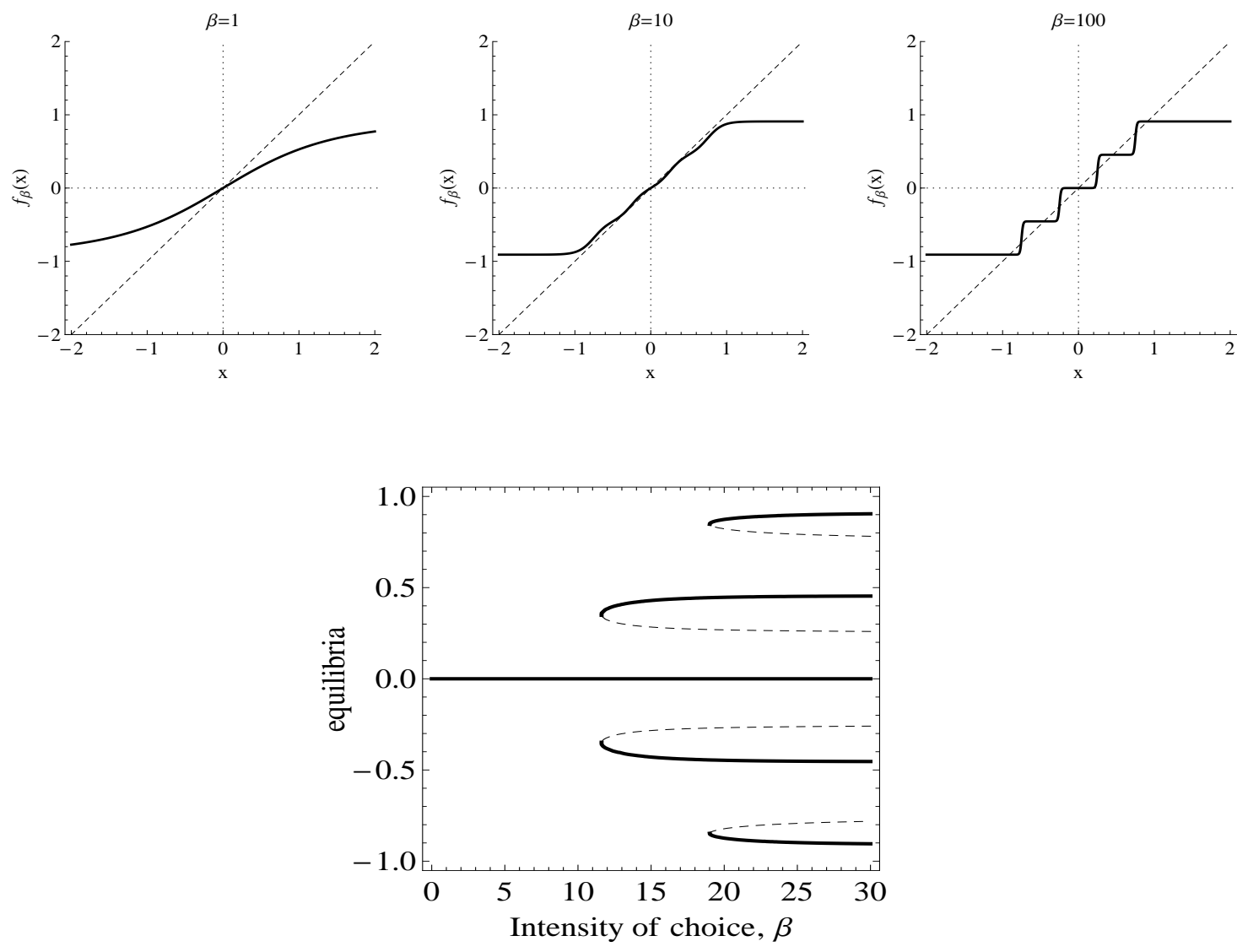

Figure 6: Top panels: The map $f_{\beta}$ in the system with 5 belief types, $b_{h} \in$ $\{-1,-1 / 2,0,1 / 2,1\}$, for different values of $\beta$. The parameter values are $b=1$, $C=0$, and $\phi_{\pi}=1.1$. Lower panel: Bifurcation diagram for this system with the same parameter values with respect to the intensity of choice. Solid lines indicate stable equilibria and dashed lines unstable equilibria. For high values of $\beta, 9$ different steady states co-exist, 5 stable separated by 4 unstable steady states.

stant beliefs. Fig. 6 illustrates graphs of the 1-D map when there are five strategy types $b_{h} \in\{-1,-1 / 2,0,1 / 2,1\}$, the costs $C$ of the fundamental predictor are low, and the monetary policy rule is such that multiple equilibria exist but the RE steady state remains locally stable. We also show the creation of five multiple steady state equilibria as the intensity of choice increases by means of the bifurcation diagram.

For small and medium values of $\beta$ the bifurcation scenario is similar to the three types case. However for high values of the intensity of choice, four additional steady states, two stable and two unstable, are created. The intuition for the appearance of the new stable steady states is similar as before. Any available predictor would give the most precise forecast if the past inflation rate is sufficiently close to it. 
A high intensity of choice causes a large group of agents to choose this successful predictor, locking the inflation dynamics into a self-fulfilling stable equilibrium steady state close to that predictor. One can construct similar examples for any finite (odd) number, $H=2 K+1$, of forecasting strategies generating $H$ multiple stable equilibria. A finite class of forecasting rules seems reasonable as boundedly rational agents may exhibit "digit preference" and restrict their inflation predictions to values in integer numbers, e.g., $2 \%, 3 \%$, or to half percentages, e.g., $2.5 \%$ or $3.5 \%$, within the range of historically observed values from say $-5 \%$ to $+15 \%^{11}$

The results in this section show that, in the presence of few belief types and evolutionary selection between different predictors, the Taylor principle is no longer sufficient to guarantee uniqueness and stability of the RE steady state. As a concrete example, the reaction coefficient suggested by Taylor (1993), i.e., $\phi_{\pi}=1.5$ might not be sufficient to guarantee the determinacy (uniqueness and stability) of the RE equilibrium (see Figs. 3 and 5).

\subsection{Many Belief Types}

The previous analysis shows that in an economy with an ecology of 3 or 5 fundamentalists and biased beliefs, a cumulative process leading to accelerating inflation or deflation does not arise. Rather, for high intensity of choice and depending on the strength of policy reactions to inflation, the system might lock in one of multiple steady state equilibria, with a majority of agents using the forecasting rule with the smallest error at that equilibrium steady state. A natural question is: what happens when the number of constant forecasting rules increases and approaches infinity? As we will see, if agents select beliefs from a continuum of forecasting rules, representing an ecology containing all constant predictions, the cumulative

\footnotetext{
${ }^{11}$ Digit preference has been observed in both survey measures of expectations and experimental data. Curtin (2005) and Duffy and Lunn (2009) find evidence for digit preference respectively in the Michigan Survey data and in the EU Consumer Survey for Ireland. Assenza, Heemeijer, Hommes, and Massaro (2011) observed digit preference in learning-to-forecast-experiments adopting the New Keynesian model. In particular they find that about $14 \%$ of predictions of participants are integers and about $32 \%$ of predictions have a precision of 1 decimal. Overall, about $99.6 \%$ of predictions have a maximum precision of 2 decimals.
} 
process will reappear when the policy rule does not satisfy the Taylor principle.

Suppose there are $H$ belief types $b_{h}$, all available at zero costs. The evolutionary dynamics with $H$ belief types is given by

$$
\pi_{t}=\frac{1}{\phi_{\pi}} \cdot \frac{\sum_{h=1}^{H} b_{h} e^{-\beta\left(\pi_{t-1}-b_{h}\right)^{2}}}{\sum_{h=1}^{H} e^{-\beta\left(p i_{t-1}-b_{h}\right)^{2}}}=: f_{\beta}^{H}\left(\pi_{t-1}\right)
$$

The dynamics of the system with $H$ belief types $b_{h}$ is described by a $1-\mathrm{D}$ map $f_{\beta}^{H}$. What can be said about the dynamical behavior when $H$ is large? In general, it is difficult to obtain analytical results for systems with many belief types. We apply the concept of Large Type Limit (LTL henceforth) introduced in Brock, Hommes, and Wagener (2005) to approximate the evolutionary system with many belief types in (3.5). Suppose that at the beginning of the economy, i.e., at period $t=0$, all $H$ belief types $b=b_{h} \in \mathbb{R}$ are drawn from a common initial distribution with density $\psi(b)$. We can derive the LTL of the system as follows. Divide both numerator and denominator of (3.5) by $H$ and rewrite the " $H$-type system" as

$$
\pi_{t}=\frac{1}{\phi_{\pi}} \cdot \frac{\frac{1}{H} \sum_{h=1}^{H} b_{h} e^{-\beta\left(\pi_{t-1}-b_{h}\right)^{2}}}{\frac{1}{H} \sum_{h=1}^{H} e^{-\beta\left(\pi_{t-1}-b_{h}\right)^{2}}} .
$$

The LTL is obtained by replacing the sample mean with the population mean in both the numerator and the denominator, yielding

$$
\pi_{t}=\frac{1}{\phi_{\pi}} \cdot \frac{\int b e^{-\beta\left(\pi_{t-1}-b\right)^{2}} \psi(b) d b}{\int e^{-\beta\left(\pi_{t-1}-b\right)^{2}} \psi(b) d b}=: F_{\beta}\left(\pi_{t-1}\right)
$$

As shown in Brock, Hommes, and Wagener (2005), when the number of strategies $H$ is sufficiently large, the LTL dynamical system (3.6) is a good approximation of the dynamical system with $H$ belief types given by (3.5). In particular, if $H$ is large then with high probability the steady states and their local stability conditions coincide for both the LTL map $F_{\beta}$ and the $H$-belief system map $f_{\beta}^{H}$. In other words, properties of the evolutionary dynamical system with many types of 

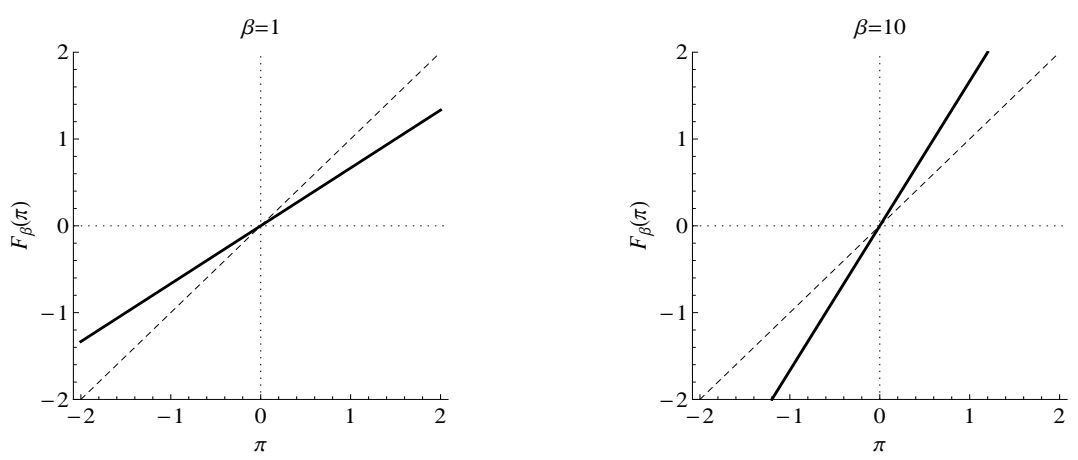

Figure 7: Graphs of the LTL map $F_{\beta}$ in (3.7) when the Taylor principle is not satisfied for a normal distribution $\psi(b) \simeq N(0,0.25)$ of initial beliefs. The reaction coefficient is $\phi_{\pi}=0.5$. Left: $\beta=1$. Right: $\beta=10$.

agents can be studied using the LTL system.

For suitable distributions $\psi(b)$ of initial beliefs, the LTL (3.6) can be computed explicitly. As an illustrative example consider the case when $\psi(b)$ is a normal distribution, $\psi(b) \simeq N\left(m, s^{2}\right)$. Plugging the normal density in (3.6), a straightforward computation shows that the LTL map $F_{\beta}$ is linear with slope increasing in $\beta$, given by

$$
F_{\beta}(\pi)=\frac{1}{\phi_{\pi}} \cdot \frac{m+2 \beta s^{2} \pi}{1+2 \beta s^{2}}
$$

In particular, when the initial beliefs distribution is centered around $m=0$, the unique steady state of the LTL map is the RE equilibrium, $\pi^{*}=0$. This case is illustrated in Fig. 7, where we show the LTL map for different values of the intensity of choice when the interest rate rule does not satisfy the Taylor principle.

For $\beta=\beta^{*}=\frac{\phi_{\pi}}{2 s^{2}\left(1-\phi_{\pi}\right)}$ the slope of the linear map is exactly 1 . Hence, the $\mathrm{RE}$ equilibrium is globally stable for $\beta<\beta^{*}$ and unstable otherwise. When $m \neq 0$, i.e., when the initial belief distribution is not symmetric with respect to the fundamental equilibrium, the unique steady state of the LTL system is not the REE, but the stability result and critical value $\beta^{*}$ do not change (cf. footnote 9 ).

We can conclude, therefore, that when initial beliefs are drawn from a normal distribution centered around the REE and the number of belief types is sufficiently 
high, an increase in the intensity of choice, beyond the bifurcation value $\beta^{*}$, leads to instability of the system. Indeed, when $\beta$ is low, agents are more or less equally distributed among predictors. This means that the average expected inflation will be close to zero. Hence realized inflation will be close to the steady state value, more agents will adopt the steady state predictor and inflation will converge. However, when the intensity of choice increases and agents can switch faster to better predictors, the system becomes unstable. This is so because, for example, when the inflation rate is above its steady state value most agents will switch to an even more positive bias belief, leading to an even higher realized inflation rate. A cumulative process of ever increasing inflation arises again.

Note that increasing the variance $s^{2}$ of the normal distribution of initial beliefs has exactly the same effect on the LTL dynamics (3.7) as increasing the intensity of choice. When $\phi_{\pi}<1$ we have that for $s^{2}<\frac{\phi_{\pi}}{2 \beta\left(1-\phi_{\pi}\right)}$ the LTL map is globally stable, and it is unstable otherwise. Hence, when many initial beliefs are drawn from a normal distribution with small variance, the system will be stable, otherwise it will be unstable and a cumulative process will arise. The spread of initial beliefs is therefore an important element for the stability of the economy.

In the previous example we have assumed a normal distribution $\psi(b)$ of initial beliefs. Applying the results derived in Hommes and Wagener (2010), similar conclusions can be obtained for general distribution functions of initial beliefs. In fact, for systems with many belief types $b_{h}$ and initial beliefs drawn from a fixed strictly positive distribution function, when the intensity of choice becomes sufficiently large, a cumulative process arises with high probability. ${ }^{12}$

To get some intuition for this result, it will be instructive to look at the limiting case $\beta=\infty$. When there is a continuum of beliefs, the best predictor in every period, according to past forecast error, will be the predictor that exactly coincides with last period's inflation realization, $b_{h}=\pi_{t-1}$. For $\beta=\infty$, all agents will switch

\footnotetext{
${ }^{12}$ This result follows by applying Lemma 1, p. 31 of Hommes and Wagener (2010), stating that for any strictly positive distribution function $\psi$ describing initial beliefs, as the intensity of choice goes to infinity, the corresponding LTL map converges to a linear map with slope $1 / \phi_{\pi}$.
} 
to the optimal predictor. Hence, for $\beta=\infty$, the economy with heterogeneous agents updating their beliefs through reinforcement learning behaves exactly the same as an economy with a representative naive agent, for which we have shown that a cumulative process will arise when the monetary policy rule does not satisfy the Taylor principle (see Section 2, eq. (2.4)).

Finally consider a monetary authority implementing an interest rate rule that obeys the Taylor principle, i.e., $\phi_{\pi}>1$. It should be clear that the "unstable" situation shown in the right panel of Fig. 7 cannot occur. Indeed, in this case we will have that

$$
\lim _{\beta \rightarrow \infty} F_{\beta}(\pi)=\frac{1}{\phi_{\pi}} \pi
$$

Hence an interest rate rule that responds aggressively to actual inflation, i.e., $\phi_{\pi}>$ 1 , will fully stabilize the system, for all values of the intensity of choice $\beta$. In contrast, if the policy rule of the Central Bank is not sufficiently aggressive, i.e., $\phi_{\pi}<1$, then inflation dynamics will only be stable for small values of the intensity of choice, but the cumulative process will reappear when the intensity of choice is large. Therefore, when the model is indeterminate under rational expectations, there is a cumulative process for both the representative agent adaptive learning specification and the heterogeneous expectations case with many belief types. The same result holds for a normal initial distribution of beliefs centered around $m \neq 0$, even though the steady state of the dynamics will differ from the RE equilibrium in this case.

\section{Stochastic Simulations}

In this section we discuss stochastic simulations of our nonlinear model with heterogeneous expectations in order to match some characteristics of US inflation quarterly data over the period 1960-2007. We consider an ecology of $H=12$ 

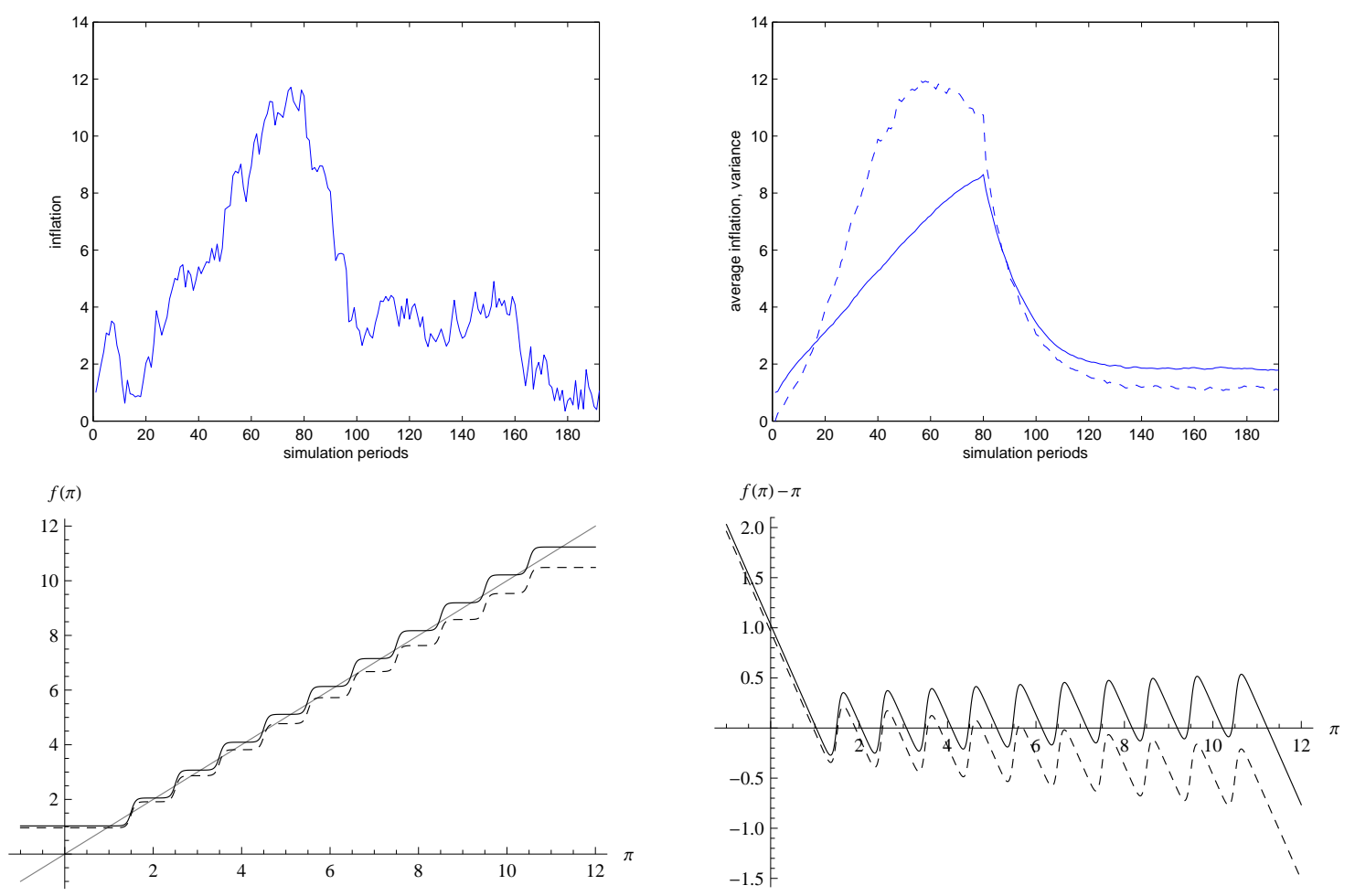

Figure 8: Top Panels: Simulated inflation time series. Left: Simulated inflation for a particular realization of the stochastic shocks. Right: Average inflation (solid line) and its variance (dashed line) over 1000 simulations. Bottom Panels: Steady states of the dynamics before and after the structural break. Left: The steady states of the dynamics as intersection points of the 45-degree line with the map $f_{0.98}^{H}(\pi)$ before the structural break (solid) and the map $f_{1.05}^{H}(\pi)$ after the structural break (dashed). Right: Plot of the maps $f_{\phi_{\pi}}^{H}(\pi)-\pi$. The same steady states are now clearly visible as intersections with the horizontal axis. The stable (unstable) steady states are marked with black (white) dots. The basin of attraction of a stable steady state is the interval between two adjacent unstable steady states.

forecasting rules, $b_{h} \in\{1, \ldots, 11\},{ }^{13}$ so that the dynamics of inflation is given by

$$
\pi_{t}=f_{\phi_{\pi}}^{H}\left(\pi_{t-1}\right)+\varepsilon_{t},
$$

\footnotetext{
${ }^{13}$ The constant forecasting rules considered are within the range of integer predictors described by Curtin (2005) in the Michigan Survey data on inflation expectations.
} 
where the map $f_{\phi_{\pi}}^{H}$ is defined as ${ }^{14}$

$$
f_{\phi_{\pi}}^{H}\left(\pi_{t-1}\right)=\frac{1}{\phi_{\pi}} \cdot \frac{\sum_{h=1}^{H} b_{h} e^{-\beta\left(\pi_{t-1}-b_{h}\right)^{2}}}{\sum_{h=1}^{H} e^{-\beta\left(\pi_{t-1}-b_{h}\right)^{2}}}+c,
$$

and the exogenous random shocks $\varepsilon_{t}$ are drawn from a normal distribution with mean 0 and standard deviation $\sigma_{\varepsilon}=0.5$. The notation $f_{\phi_{\pi}}^{H}\left(\pi_{t-1}\right)$ stresses the fact that the nonlinear map depends on the monetary policy parameter $\phi_{\pi}$, the coefficient in the Taylor rule. In all stochastic simulations there is a structural break in period $T=80$ in the Central Bank's reaction function. In the first part of the simulations the policy rule reacts weakly to inflation (i.e., $\phi_{\pi}<1$ ), while in the second part the interest rate reacts more than point to point to inflation (i.e., $\phi_{\pi}>1$ ). In particular we consider a reaction coefficient $\phi_{\pi}=0.98$ for periods $t=1-79$ and a reaction coefficient $\phi_{\pi}=1.05$ for periods $t=80-192 .^{15}$

The stochastic time series in Figure 8 replicates the observed pattern of a strong rise in US inflation until 1980 and a sharp decline and stabilization of inflation thereafter (see also Fig. 1). Of course the particular realization shown in the top left panel is affected by stochastic shocks, but this pattern is quite common and reproduced by the time series of average inflation, averaged over 1000 stochastic simulations, in Figure 8 (top right panel). The plot of the corresponding variance of the stochastic simulations shows that the variance is low after the structural break, implying that the strong decline in inflation after the structural break is a robust feature of the nonlinear model with heterogeneous beliefs and a monetary authority that obeys the Taylor principle. On the other hand, before the structural break the variance of the stochastic simulations is large, showing that the rise in inflation can be either slow or fast depending upon the realizations of the exogenous stochastic shocks. In particular, a few large positive shocks to inflation, such as

\footnotetext{
${ }^{14}$ The presence of constant $c$ in map (4.1) stems from the fact that, in general, the constant term in the Taylor rule (2.2) might differ from the equilibrium interest rate $r$. In our simulations we assume a Taylor rule of the form $i_{t}=\phi_{\pi} \pi_{t}$, so that $c=r / \phi_{\pi}$. Assuming a standard discount factor $\delta=0.99$ we have that $c \approx 0.01 / \phi_{\pi}$.

${ }^{15}$ The value of $\phi_{\pi}$ influences the number of steady states. The number of steady states increases with $\phi_{\pi}$ when $\phi_{\pi}<1$, and it decreases with $\phi_{\pi}$ when $\phi_{\pi}>1$.
} 
large oil shocks, may trigger an increase in inflation which then becomes amplified by evolutionary pressure of self-fulfilling forecasting rules predicting high inflation.

The bottom panel in Figure 8 illustrates how the number of steady states in the nonlinear model with heterogeneous expectations changes when the monetary policy coefficient $\phi_{\pi}$ increases from 0.98 to 1.05 . Before the structural break there are 21 steady states, 11 stable ones separated by 10 unstable steady states, ranging approximately from a low level of 1 to a high level of 11. A careful look at Figure 8 (bottom right panel) reveals an important asymmetry in the basins of attraction of each stable steady state: the basin of attraction (whose endpoints consist of the two neighboring unstable steady states) is relatively large to the left of the stable steady state and relatively small to the right. In the presence of (symmetric) stochastic shocks to inflation, jumps to the basin of attraction of a higher stable steady states are therefore more likely than jumps to a lower level. This explains why for $\phi_{\pi}=0.98$ on average inflation will rise from low levels to high levels as shown by the average inflation of the stochastic simulations.

After the structural break, when the Central Bank switches to a more aggressive Taylor rule, the number of steady states has decreased from 21 to 11, with 6 stable ones separated by 5 unstable steady states, ranging from approximately 1 to 6 . Hence, an increase of the monetary policy parameter $\phi_{\pi}$ causes a number of high level steady states to disappear, ${ }^{16}$ implying more stable inflation dynamics in the stochastic nonlinear system as illustrated in the stochastic simulations after the structural break.

It is interesting to note that similar results occur when we allow for (infinitely) many constant prediction rules. Indeed our results concerning the LTL system in (3.6) in Section 3.2 show that, when agents are sensitive to difference in forecasting performance (i.e., for high values of the intensity of choice $\beta$ ), the inflation dynamics with an ecology of many steady state predictors drawn from a normal distribution

\footnotetext{
${ }^{16} \mathrm{As} \phi_{\pi}$ increases from 0.98 to 1.05 the high level steady states disappear in pairs of two (one stable and one unstable) through a number of subsequent saddle-node bifurcations.
} 
of initial beliefs is well approximated by the linear map in (3.8), with slope $\frac{1}{\phi_{\pi}}$. This implies globally stable inflation dynamics approaching the RE equilibrium rate of inflation when $\phi_{\pi}>1$, but exploding inflation dynamics when $\phi_{\pi}<1$. Hence, in an ecology with many steady state predictors when the Central Bank uses a Taylor rule with $\phi_{\pi}<1$ a cumulative process of rising inflation is very likely, while the monetary authority can manage heterogeneous expectations and achieve global macro economic stability by using a more aggressive Taylor rule with $\phi_{\pi}>1$.

\section{Concluding Remarks}

We have used a simple frictionless DSGE model to study the role of heterogeneous expectations about future inflation and the potential (de-)stabilizing effect of different interest rate rules. We use the heterogeneous expectations framework of Brock and Hommes (1997), where the ecology of forecasting rules is disciplined by endogenous, evolutionary selection of strategies with agents switching towards more successful rules.

Macroeconomic stability and inflation dynamics depend in interesting ways on the set of forecasting strategies and the coefficient of an interest rate rule à la Taylor. When the monetary authority responds weakly to inflation, heterogeneous agents trying to learn from their forecast errors receive misleading signals from the market. Instead of leading the economy closer to the equilibrium, these signals cause a cumulative process of rising inflation, triggered by exogenous shocks to economic fundamentals and reinforced by self-fulfilling expectations of high inflation. In contrast, when the nominal interest rate is adjusted more than point for point in response to inflation, the monetary authority can manage heterogeneous expectations by sending signals that help agents to correct their forecast errors instead of compounding them. The rationale for an aggressive monetary policy is therefore rather different in the case of heterogeneous beliefs compared to the homogeneous rational expectation case. In presence of heterogeneous expectations, 
by reacting aggressively to inflation, the Central Bank sends correct signals for the evolutionary selection of strategies and induces stable dynamics converging to the RE steady state. Under rational expectations, by obeying the Taylor principle, the monetary authority induces dynamics that will explode in any equilibrium but one. Ruling out explosive paths guarantees then uniqueness of the equilibrium. ${ }^{17}$

However, while the Taylor principle is sufficient to ensure convergence to the RE steady state in the case of a continuum of beliefs, the standard policy recommendation, i.e., $\phi_{\pi}>1$, is no longer sufficient to guarantee uniqueness and global stability of the RE steady state in the case of finitely many belief types. In fact, in order to avoid multiple equilibria, the policy rule must be aggressive enough (e.g., $\phi_{\pi}>\phi_{\pi}^{a} \geq 2$ in the 3 belief types example with small costs of Section 3.1) to ensure that realized inflation is sufficiently close to the RE steady state, so that the fundamental predictor will perform relatively better than other strategies in the economy. The intuition is that with a continuum of beliefs and any $\phi_{\pi}>1$, there is always a belief type closer to the fundamental than current inflation performing better, thus pushing inflation gradually towards the fundamental. In contrast, with finitely many types and $\phi_{\pi}>1$, but not large enough (i.e., $\phi_{\pi}<\phi_{\pi}^{a}$ ), the system may get locked in an almost self-fulfilling steady state belief. We would like to stress that the case of finitely many belief types seems empirically relevant as digit preference has been observed in experimental and survey data (see e.g., Assenza, Heemeijer, Hommes, and Massaro (2011), Curtin (2005) and Duffy and Lunn (2009)) and as our stochastic simulations illustrate.

Future work should further investigate the effect of heterogeneous expectations on the dynamics of aggregate output and inflation in models with frictions and nominal rigidities, such as the New Keynesian framework, and the conditions under which monetary policy rules may stabilize or may fail to stabilize aggregate macroeconomic variables.

In a recent paper Branch and McGough (2010) already studied heterogeneous

\footnotetext{
${ }^{17}$ See Cochrane (2010) for a discussion.
} 
expectations in the New Keynesian model. However, they only present numerical simulations showing instability and complex dynamics. An interesting topic for future work would be, for example, to apply our analytical framework to the fullyfledged New Keynesian model setting. 


\section{APPENDIX}

\section{A Model with a finite number of types}

In this appendix we consider the dynamics of the general model with $H$ types, as introduced in the beginning of Section 3. The dynamics are given by

$$
\pi_{t}=\frac{1}{\phi_{\pi}} \cdot \frac{\sum_{h=1}^{H} b_{h} \exp \left(\beta U_{h, t-1}\right)}{\sum_{h=1}^{H} \exp \left(\beta U_{h, t-1}\right)}=\frac{1}{\phi_{\pi}} \cdot \frac{\sum_{h=1}^{H} b_{h} \exp \left(-\beta\left[\left(\pi_{t-1}-b_{h}\right)^{2}+C_{h}\right]\right)}{\sum_{h=1}^{H} \exp \left(-\beta\left[\left(\pi_{t-1}-b_{h}\right)^{2}+C_{h}\right]\right)} .
$$

Recall that agents can choose one of the $H$ forecasting rules $\widehat{E}_{t, h} \pi_{t+1}=b_{h}$ available at cost $C_{h}$. The reaction coefficient $\phi_{\pi}>0$ in the interest rate rule (2.2) measures the aggressiveness of the monetary policy.

The map $\pi_{t}=f\left(\pi_{t-1}\right)$, where $f$ denotes the RHS of (A.1), defines a one dimensional dynamical system, whose properties are described in the following technical Lemma.

Lemma 1. Let $f: \mathbb{R} \rightarrow \mathbb{R}$ describe the dynamics of the system with finite number of types. Let $\underline{b}=\min _{h}\left\{b_{h}\right\}$ and $\bar{b}=\max _{h}\left\{b_{h}\right\}$ denote the smallest and the largest available forecasts. Then:

1. For $\beta>0$ function $f$ is strictly increasing.

2. $f(x) \rightarrow \underline{b} / \phi_{\pi}$ for $x \rightarrow-\infty$ and $f(x) \rightarrow \bar{b} / \phi_{\pi}$ for $x \rightarrow \infty$.

Proof. To show that the map $f$ is increasing we use the same strategy as in Hommes and Wagener (2010). Multiplying both numerator and denominator by $\exp \left(-\beta \pi_{t-1}^{2}\right)$, the map can be rewritten as

$$
f\left(\pi_{t-1}\right)=\frac{1}{\phi_{\pi}} \cdot \frac{\sum_{h=1}^{H} b_{h} \exp \left(-\beta\left[-2 \pi_{t-1} b_{h}+b_{h}^{2}+C_{h}\right]\right)}{\sum_{h=1}^{H} \exp \left(-\beta\left[-2 \pi_{t-1} b_{h}+b_{h}^{2}+C_{h}\right]\right)} .
$$

We write $x_{h}:=\exp \left(-\beta\left[-2 \pi_{t-1} b_{h}+b_{h}^{2}+C_{h}\right]\right)$, introduce the normalization factor $Z=\sum_{h=1}^{H} x_{h}$, and notice that $n_{h, t}=x_{h} / Z$. Then the derivative $f^{\prime}$ is given by

$$
\begin{aligned}
f^{\prime}\left(\pi_{t-1}\right) & =\frac{1}{\phi_{\pi}} \cdot \sum_{h=1}^{H} \frac{2 \beta b_{h}^{2} y_{h} Z-b_{h} y_{h} \sum_{h=1}^{H} 2 \beta b_{h} y_{h}}{Z^{2}}= \\
& =\frac{2 \beta}{\phi_{\pi}} \cdot\left(\sum_{h} \frac{b_{h}^{2} x_{h}}{Z}-\sum_{h} \frac{b_{h} x_{h}}{Z} \sum_{h} \frac{b_{h} x_{h}}{Z}\right)= \\
& =\frac{2 \beta}{\phi_{\pi}} \cdot\left(\sum_{h} b_{h}^{2} n_{h, t}-\left(\sum_{h} b_{h} n_{h, t}\right)^{2}\right) .
\end{aligned}
$$

The term in the last brackets is positive since it can be interpreted as the variance of a discrete stochastic variable $\xi$ taking values $b_{h}$ with probability $n_{h, t}$. Thus, $f^{\prime}\left(\pi_{t-1}\right)>0$ for $\beta>0$, i.e., 
function $f$ is strictly increasing.

When $\pi_{t-1}$ is large enough, the forecasting rule with $\widehat{E}_{t-2} \pi_{t-1}=\bar{b}$ has the highest performance measure, because the squared error term dominates constant costs. Hence, as $\pi_{t-1} \rightarrow \infty$, asymptotically all the agents use this forecasting rule, i.e., $n_{\bar{b}} \rightarrow 1$, while the fractions of all the other rules converge to 0 . Since $f\left(\pi_{t-1}\right)=\sum_{h} b_{h} n_{h, t} / \phi_{\pi}$, we obtain that $f\left(\pi_{t-1}\right) \rightarrow \bar{b} / \phi_{\pi}$ for $\pi_{t-1} \rightarrow \infty$. The proof that $f\left(\pi_{t-1}\right) \rightarrow \underline{b} / \phi_{\pi}$ for $\pi_{t-1} \rightarrow-\infty$ is similar.

This Lemma implies that the dynamics (A.1) are quite simple. Independently of the initial condition, they monotonically converge to one of the finite number of steady states. For the generic case in which there are only hyperbolic steady states (i.e., no steady state $\pi^{*}$ exists with $\left.f^{\prime}\left(\pi^{*}\right)=1\right)$ the number of steady states is odd and the locally stable steady states alternate with the unstable steady states. The basin of attraction of a stable steady state is the largest possible interval containing a given steady state without any other steady states, see illustration in the lower left panel of Fig. 8. Furthermore, the dynamics are bounded within the interval $\left(\underline{b} / \phi_{\pi}, \bar{b} / \phi_{\pi}\right)$.

\section{B Dynamics of the model with 3 types}

This appendix investigates the global dynamics of the 3-type system considered in Section 3.1. We refer the reader to Kuznetsov (1995) for a detailed mathematical treatment of bifurcation theory.

With 3 forecasting rules, $\widehat{E}_{1, t} \pi_{t+1}=0, \widehat{E}_{2, t} \pi_{t+1}=b$ and $\widehat{E}_{3, t} \pi_{t+1}=-b$, where $b>0$ is the bias parameter, system (A.1) becomes $\pi_{t}=f_{\beta}\left(\pi_{t-1}\right)$, where

$$
f_{\beta}(\pi)=\frac{b}{\phi_{\pi}} \cdot \frac{e^{-\beta(\pi+b)^{2}}+e^{-\beta(\pi-b)^{2}}}{e^{-\beta\left(\pi^{2}+C\right)}+e^{-\beta(\pi+b)^{2}}+e^{-\beta(\pi-b)^{2}}}=\frac{b}{\phi_{\pi}} \cdot \frac{1-e^{-4 \beta b \pi}}{1+e^{-4 \beta b \pi}+e^{-\beta\left(C-b^{2}+2 b \pi\right)}} .
$$

The following result will be useful.

Lemma 2. Equation $2+e^{x}-x e^{x}=0$ has a unique solution $x^{*} \in(1,2)$. For $x<x^{*}$ we have $2+e^{x}-x e^{x}>0$ and for $x>x^{*}$ we have $2+e^{x}-x e^{x}<0$.

Proof. Consider function $g(x)=2+e^{x}-x e^{x}$. Notice that $\lim _{x \rightarrow-\infty} g(x)=2, \lim _{x \rightarrow \infty} g(x)=-\infty$, $g(0)=3$, and that derivative $g^{\prime}(x)=-x e^{x}$. Hence, for $x \leq 0$ function $g$ increases from 2 to 3 and has no zeros. For $x>0$ function $g$ is strictly decreasing and has at most one zero. On the other hand, $g(1)=2>0$, while $g(2)=2-e^{2}<0$, because $e^{x}>1+x$ for $x=2$ becomes $e^{2}>3$. Applying the intermediate value theorem we obtain that there exists $x^{*}$, zero of function $g$, and that $x^{*} \in(1,2)$. 
We proceed as follows. First, we derive two useful and important results. In Proposition B.1 we analyze the local stability of the RE steady state, while in Proposition B.2 we derive the dynamics for the limiting case $\beta=+\infty$. These results will allow us to distinguish between the cases of high and low costs, and, in the latter case, between weak, moderate and aggressive monetary policy. Second, we study the concavity of function $f_{\beta}$ in Lemma 3 . Combining it with Proposition B.1 we will then be able to give a full characterization of dynamics for the high cost case and to prove Proposition 3.4. Third, inspired by Proposition B.2, we formalize in Lemma 4 the intuition that whenever non-fundamental steady states exist for $\beta=+\infty$, they will also exist for $\beta$ high enough. Combining this Lemma with results of Proposition B.1 we will derive Propositions 3.1 and 3.2. Finally, we will prove the important result of Proposition 3.3 on global stability in the low cost case with aggressive monetary policy.

The following result gives the conditions for the local stability of the RE steady state.

Proposition B.1 (Local stability of the RE steady state). Consider the dynamics given by (B.1). Let $x^{*}$ denote the unique solution of equation $2+e^{x}-x e^{x}=0$. The following cases are possible:

(1) $C \geq b^{2}$ ("high costs"). Then there exists a positive value $\beta^{*}$, such that for $\beta<\beta^{*}$ the $R E$ steady state is locally stable, and for $\beta>\beta^{*}$ the RE steady state is unstable.

(2) $C<b^{2}$ ("low costs"). Then two cases are possible:

(2a) when $\phi_{\pi}<2\left(x^{*}-1\right) \frac{b^{2}}{b^{2}-C}$ two values $0<\beta_{1}^{*}<\beta_{2}^{*}$ exist such that for $\beta \notin\left[\beta_{1}^{*}, \beta_{2}^{*}\right]$ the $R E$ steady state is locally stable, and for $\beta \in\left(\beta_{1}^{*}, \beta_{2}^{*}\right)$ the RE steady state is unstable.

(2b) when $\phi_{\pi}>2\left(x^{*}-1\right) \frac{b^{2}}{b^{2}-C}$ the RE steady state is locally stable for any $\beta \geq 0$.

Proof. The fractions of three rules in the RE steady state are given by $n_{1}^{*}=e^{-\beta C} / Z$ and $n_{2}^{*}=$ $n_{3}^{*}=e^{-\beta b^{2}} / Z$, where $Z=e^{-\beta C}+2 e^{-\beta b^{2}}$. Substituting these fractions into (A.2), we find the derivative of map $f_{\beta}$ in the $\mathrm{RE}$ steady state

$$
f_{\beta}^{\prime}(0)=\frac{4 b^{2} \beta}{\phi_{\pi}} \cdot \frac{e^{-\beta b^{2}}}{2 e^{-\beta b^{2}}+e^{-\beta C}}=\frac{4 b^{2} \beta}{\phi_{\pi}} \cdot \frac{1}{2+e^{-\beta\left(C-b^{2}\right)}}
$$

The condition of local stability is given by $f_{\beta}^{\prime}(0)<1$, or, equivalently by $h(\beta)<\phi_{\pi}$, where function $h$ is defined as

$$
h(\beta)=\frac{4 b^{2} \beta}{2+e^{-\beta\left(C-b^{2}\right)}} .
$$

Notice that $h(0)=0$ and the derivative of the function in $\beta$ is given by

$$
h^{\prime}=\frac{4 b^{2}}{\left(2+e^{-\beta\left(C-b^{2}\right)}\right)^{2}}\left(2+e^{-\beta\left(C-b^{2}\right)}+\beta\left(C-b^{2}\right) e^{-\beta\left(C-b^{2}\right)}\right)=\frac{4 b^{2}}{\left(2+e^{x}\right)^{2}}\left(2+e^{x}-x e^{x}\right),
$$


where we introduced the variable $x=\left(b^{2}-C\right) \beta$.

In the high costs case, $C \geq b^{2}$, variable $x$ is negative and, according to Lemma 2, function $h$ strictly increases in $\beta$ from 0 to $\infty$. Thus, when $\beta$ becomes higher than the bifurcation value $\beta^{*}$ defined as $\beta^{*}=h^{-1}\left(\phi_{\pi}\right)$, the RE steady state looses stability.

If $C<b^{2}$, variable $x$ is positive and changes from 0 to $\infty$ together with $\beta$. We have then that function $h$ is initially increasing in $\beta$ and then decreasing. Function $h$ takes its maximum value in the point where $x=x^{*}$, i.e., when $\beta=x^{*} /\left(b^{2}-C\right)$. The value of function $h$ in this point is given by

$$
h\left(\frac{x^{*}}{b^{2}-C}\right)=\frac{4 b^{2}}{2+e^{x^{*}}} \cdot \frac{x^{*}}{b^{2}-C}=\frac{4 b^{2}}{2+\frac{2}{x^{*}-1}} \cdot \frac{x^{*}}{b^{2}-C}=\left(x^{*}-1\right) \frac{2 b^{2}}{b^{2}-C} .
$$

The maximum value of $h$ is positive according to Lemma 2. If it is larger than $\phi_{\pi}$, then the two solutions of equation $h(\beta)=\phi_{\pi}$ define an interval $\left(\beta_{1}, \beta_{2}\right)$ where $h(\beta)>\phi_{\pi}$, and so the RE steady state is unstable. In the opposite case, if the maximum value of $h$ is smaller than $\phi_{\pi}$, then $h(\beta)<\phi_{\pi}$ for any $\beta$ and the RE steady state is always locally stable.

Proposition B.2 (Steady states for $\beta=+\infty$ ). Consider the dynamics given by (B.1) for the special case of $\beta=+\infty$. Let us denote $\pi^{*}=0, \pi^{+}=b / \phi_{\pi}$ and $\pi^{-}=-b / \phi_{\pi}$. The following cases are possible:

(1) $C \geq b^{2}$ ("high costs"). Then there are two locally stable steady states, $\pi^{+}$and $\pi^{-}$, with corresponding basins of attraction $(0, \infty)$ and $(-\infty, 0)$. The RE steady state $\pi^{*}$ is unstable.

(2) $C<b^{2}$ ("small costs"). Then two cases are possible:

(2a) when $\phi_{\pi}<\frac{2 b^{2}}{b^{2}-C}$ the system has three locally stable steady states, $\pi^{*}, \pi^{+}$and $\pi^{-}$. The basin of attraction of the RE steady state is $\left(-\frac{b^{2}-C}{2 b}, \frac{b^{2}-C}{2 b}\right)$.

(2b) when $\phi_{\pi}>\frac{2 b^{2}}{b^{2}-C}$ there exists a unique, globally stable RE steady state.

Proof. We will derive the map $f_{\infty}$ governing the dynamics in case $\beta=\infty$ explicitly. In this case the best performing rule will be chosen by all population at any period. The performances of three rules are given by

$$
U_{1, t-1}=-\pi_{t-1}^{2}-C, \quad U_{2, t-1}=-\pi_{t-1}^{2}+2 b \pi_{t-1}-b^{2}, \quad U_{3, t-1}=-\pi_{t-1}^{2}-2 b \pi_{t-1}-b^{2} .
$$

Assume, first, that $\pi_{t-1}>0$. Then the second rule $\left(\widehat{E}_{2, t} \pi_{t+1}=b\right)$ is always better than the third one $\left(\widehat{E}_{3, t} \pi_{t+1}=-b\right)$. Comparing the second rule with the first one $\left(\widehat{E}_{1, t} \pi_{t+1}=0\right)$ we find that

$$
U_{2, t-1}>U_{1, t-1} \quad \Leftrightarrow \quad 2 b \pi_{t-1}-b^{2}>-C \quad \Leftrightarrow \quad \pi_{t-1}>\frac{b^{2}-C}{2 b}
$$


For $C<b^{2}$ the RHS of the last inequality gives a threshold after which the agents would use the non-RE forecasting rule. When $C>b^{2}$ the RE forecasting rule will never be used if $\pi_{t-1}>0$.

Analogously we find that for $\pi_{t-1}<0$ the third rule always outperforms the second one and it is better than the first if and only if $\pi_{t-1}$ is less than the threshold $-\left(b^{2}-C\right) /(2 b)$. When $C>b^{2}$ the RE forecasting rule will never be used if $\pi_{t-1}<0$.

We conclude that function $f_{\infty}$ has the following form. For $C \geq b^{2}$

$$
f_{\infty}\left(\pi_{t-1}\right)= \begin{cases}\frac{b}{\phi_{\pi}} & \text { if } \pi_{t-1}>0 \\ 0 & \text { if } \pi_{t-1}=0 \\ -\frac{b}{\phi_{\pi}} & \text { if } \pi_{t-1}<0\end{cases}
$$

whereas for $C<b^{2}$

$$
f_{\infty}\left(\pi_{t-1}\right)= \begin{cases}\frac{b}{\phi_{\pi}} & \text { if } \pi_{t-1}>\frac{b^{2}-C}{2 b} \\ 0 & \text { if } \pi_{t-1} \in\left(-\frac{b^{2}-C}{2 b}, \frac{b^{2}-C}{2 b}\right) \\ -\frac{b}{\phi_{\pi}} & \text { if } \pi_{t-1}<-\frac{b^{2}-C}{2 b}\end{cases}
$$

In the high cost case two steady states $\pi^{+}$and $\pi^{-}$always exist and function $f_{\infty}$ is flat around them. This proves part (1) of the Lemma. In the low cost case the non-RE steady state $\pi^{+}$ exists if and only if the 45-degree line has an intersection with the upper horizontal parts of $f_{\infty}$, i.e., when it intersects the line $b / \phi_{\pi}$ at some $\pi>\left(b^{2}-C\right) /(2 b)$. The condition for this is $b / \phi_{\pi}>\left(b^{2}-C\right) /(2 b)$ or, equivalently, $\phi_{\pi}<\left(2 b^{2}\right) /\left(b^{2}-C\right)$, which distinguishes cases $2(a)$ and 2(b) of Lemma. Lower non-RE steady state $\pi^{-}$exists if and only if the upper non-RE steady state $\pi^{+}$exist due to symmetry of map $f_{\infty}$.

Lemma 3. If $C \geq b^{2}$ function $f_{\beta}$ is concave on the set $(0, \infty)$ for every $\beta>0$. If $C<b^{2}$ function $f_{\beta}$ defined on $(0, \infty)$ is concave for every $0<\beta<\frac{\ln 4}{b^{2}-C}$.

Proof. We find with direct computations that the second derivative of $f_{\beta}$ is given by $f_{\beta}^{\prime \prime}(\pi)=-\frac{4 b^{3} \beta^{2}\left(1-e^{-4 b \pi \beta}\right) e^{-(C+2 b \pi) \beta}}{\phi_{\pi}\left(e^{C \beta}+e^{b(b-2 \pi) \beta}+e^{(C-4 b \pi) \beta}\right)^{3}}\left(e^{\left(b^{2}-C\right) \beta}-e^{2\left(b^{2}-C-b \pi\right) \beta}+8 e^{-2 b \pi \beta}+e^{\left(b^{2}-C-4 b \pi\right) \beta}\right)$.

The fraction in this expression is positive for $\pi>0$. The term between brackets can be rewritten as

$$
e^{\left(b^{2}-C\right) \beta}\left(1+e^{-4 b \pi \beta}\right)+e^{-2 b \pi \beta}\left(8-e^{2\left(b^{2}-C\right) \beta}\right) .
$$


In the high cost case, $C \geq b^{2}$, this expression is positive because $e^{2\left(b^{2}-C\right) \beta} \leq 1$. It implies that $f_{\beta}^{\prime \prime}(\pi)<0$ for $\pi>0$ and $\beta>0$.

Consider the low cost case, $C<b^{2}$, and fix $\beta$ such that $0<\beta<\frac{\ln 4}{b^{2}-C}$. When $\pi=0$, the term between brackets becomes $8+2 e^{\left(b^{2}-C\right) \beta}-e^{2\left(b^{2}-C\right) \beta}=8+2 x-x^{2}$, where we introduced $x=e^{\left(b^{2}-C\right) \beta}$. Notice that $1<x<4$ for a given $\beta$, and, therefore, the term is positive. Hence, by continuity of the second derivative, $f_{\beta}^{\prime \prime}<0$ for small $\pi>0$. With a further increase of $\pi$, the sign of the second derivative would change when the term in brackets is zero, i.e., when

$$
\frac{e^{\left(b^{2}-C\right) \beta}}{8-e^{2\left(b^{2}-C\right) \beta}}=-\frac{e^{-2 b \pi \beta}}{1+e^{-4 b \pi \beta}} .
$$

The left hand-side can be written as function $x /\left(8-x^{2}\right)$ and for $x \in(1,4)$ we have that the left hand-side does not take values in the interval $[-0.5,0)$. However, the right-hand side does take values only in this interval, as a function $-t /\left(1+t^{2}\right)$ of $t=e^{-2 b \pi \beta} \in(0,1]$. It means that there is no $\pi$ to satisfy equality (B.3) and $f_{\beta}^{\prime \prime}$ does not change its sign. We established that $f_{\beta}^{\prime \prime}(\pi)<0$ for a given $\beta$ and for any $\pi>0$. This completes the proof.

Proof of Proposition 3.4. The previous Lemma shows that for the high cost case, $C \geq b^{2}$, function $f_{\beta}$ is concave for $\pi>0$ irrespectively of $\beta>0$. In Proposition B.1(1) we found that there is a critical value $\beta^{*}$ such that, when $\beta<\beta^{*}$, the $\mathrm{RE}$ steady state is locally stable. For such $\beta$ the global stability then follows immediately from concavity of $f_{\beta}$ for positive $\pi$ and symmetry of this function w.r.t. $\pi=0$. When the RE steady state is unstable, concavity together with boundedness of $f$ implies that there exists a unique steady state with $\pi^{+}>0$, and that this steady state is locally stable. By symmetry, there exists also a steady state $\pi^{-}<0$, also locally stable.

We comment that in the high cost case the region of instability always exists. The threshold is defined by $\beta^{*}=h^{-1}\left(\phi_{\pi}\right)$, where $h$ is defined in (B.2). The RE steady state loses its stability at $\beta=\beta^{*}$ through pitchfork bifurcation. Proposition B.2 implies that for $\beta \rightarrow \infty$ the nonfundamental and locally stable steady states $\pi^{+}$and $\pi^{-}$converge to $b / \phi_{\pi}$ and $-b / \phi_{\pi}$, respectively. Therefore, an aggressive policy of the Central Bank (i.e., high $\phi_{\pi}$ ) reduces both the interval of instability and the deviations from the RE steady state.

\section{Low Cost Case}

Assume that we are in the low cost case, $C<b^{2}$. Let us introduce the positive quantity

$$
\phi_{\pi}^{w}(b, C)=2\left(x^{*}-1\right) \frac{b^{2}}{b^{2}-C} .
$$

When the reaction coefficient $\phi_{\pi}<\phi_{\pi}^{w}(b, C)$ we define the monetary policy as "weak". In fact in 
this case the policy rule fails to ensure even local stability of the RE steady state when $\beta$ increases (see Proposition B.1(2)). We can find numerically $x^{*} \approx 1.46$, so that $\phi_{\pi}^{w}(b, C)$ is approximately given by $0.93 \frac{b^{2}}{b^{2}-C}$. Given parameters $b$ and $C$, the Central Bank can implement an interest rate rule with $\phi_{\pi}>\phi_{\pi}^{w}(b, C)$ and keep the RE steady state locally stable.

However, as Proposition B.2(2) shows, this value of $\phi_{\pi}$ can be not sufficient for the global stability of the RE steady state. Let us introduce another positive quantity

$$
\phi_{\pi}^{a}(b, C)=\frac{2 b^{2}}{b^{2}-C}
$$

When the reaction coefficient $\phi_{\pi}>\phi_{\pi}^{a}(b, C)$ we define the monetary policy as "aggressive". According to Proposition B.2(2), in this case the RE steady state is unique and globally stable, at least for $\beta=+\infty$. Of course, $\phi_{\pi}^{w}(b, C)<\phi_{\pi}^{a}(b, C)$ (it follows from Lemma 2), and when $\phi_{\pi} \in\left(\phi_{\pi}^{w}(b, C), \phi_{\pi}^{a}(b, C)\right)$ we define the monetary policy as "moderate". It is strong enough to guarantee local stability of RE steady state for every $\beta$ but it is not sufficiently aggressive to guarantee the global stability of the RE steady state for $\beta=+\infty$.

Intuitively, when $\beta$ gets larger, the map $f_{\beta}$ in (B.1) gets closer to the piece-wise map $f_{\infty}$ derived in the proof of Proposition B.2. Therefore, when the monetary policy is weak or moderate, we expect to observe the non-RE steady states for $\beta$ high enough. The following result confirms this intuition.

Lemma 4. Suppose $C<b^{2}$ and $\phi<\phi_{\pi}^{a}(b, C)$. For high enough $\beta$, the dynamics given by (B.1) have locally stable steady states $\pi^{+}>0$ and $\pi^{-}=-\pi^{+}<0$.

Proof. We will prove the existence of $\pi^{+}$. The existence of $\pi^{-}$will then follow from the symmetry of $f_{\beta}$.

Take $\varepsilon=1 / \phi_{\pi}-1 / \phi_{\pi}^{a}(b, C)=1 / \phi_{\pi}-\left(b^{2}-C\right) /\left(2 b^{2}\right)>0$, define $\delta=\varepsilon\left(b^{2}-C\right) /(2 b)>0$ and consider set $U=\left\{\pi: \pi>\left(b^{2}-C\right) /(2 b)+\delta\right\}$. Set $U$ is bounded from below and on this set $f_{\beta}(\pi)$ converges to $f_{\infty}(\pi)=b / \phi_{\pi}$ from below when $\beta \rightarrow \infty$. Hence, for any $\pi \in U$ for sufficiently high $\beta$ it holds that

$$
f_{\beta}(\pi)>\frac{b}{\phi_{\pi}} \cdot \frac{1+\varepsilon}{1+\frac{2 b^{2}}{b^{2}-C} \varepsilon}=\frac{b}{\phi_{\pi}} \cdot \frac{1+\varepsilon}{\frac{1}{\phi_{\pi}} \frac{2 b^{2}}{b^{2}-C}}=\frac{b^{2}-C}{2 b}(1+\varepsilon)=\frac{b^{2}-C}{2 b}+\delta .
$$

Thus, increasing and bounded from above, $f_{\beta}$ maps set $U$ into itself. Therefore, there should exist a locally stable steady state within set $U$.

Proof of Proposition 3.1. According to Proposition B.1(2a) the RE steady state is locally stable for small $\beta$ but loses and again acquires its local stability through two subsequent pitchfork 


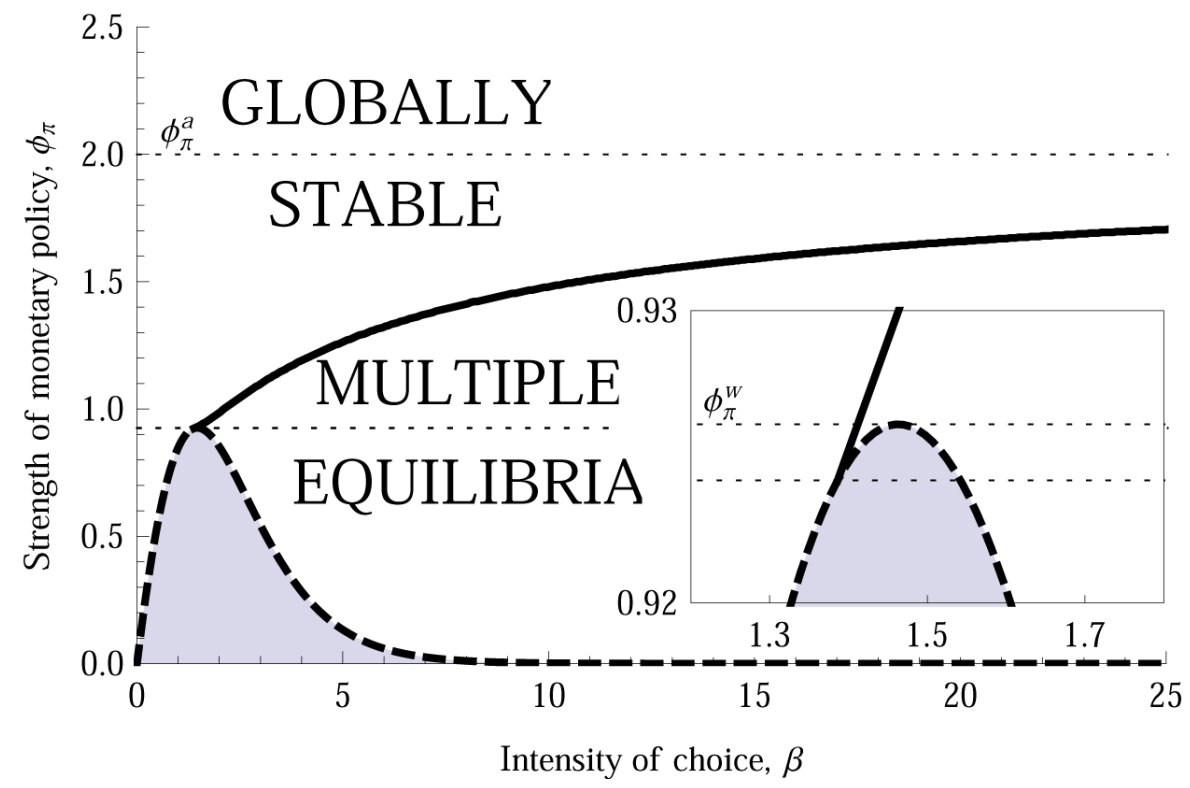

Figure 9: Bifurcation diagram of dynamics in the low cost case, shown in coordinates $\left(\beta, \phi_{\pi}\right)$. Parameters are $C=0$ and $b=1$. The dashed curve gives all the parameters of the pitchfork bifurcation, while the solid line gives the parameters of tangent bifurcation. The inset zooms the area where the curve of pitchfork bifurcation (dashed) intersects with the curve of tangent bifurcation (solid). The shaded area represents the region in the parameters space in which the $\mathrm{RE}$ is locally unstable.

bifurcations. Together with concavity of $f_{\beta}$ proved in Lemma 3, it implies global stability of RE steady state for small $\beta$. Consider now the moment of the first pitchfork bifurcation, which we denote as $\beta=\beta_{2}^{*}$. At this moment the RE steady state loses its stability, but it might do it in two different ways. If at this instance, function $f_{\beta}$ is concave for $\pi>0$, then the bifurcation at $\beta_{2}^{*}$ is supercritical and two stable non-RE steady states are created (in this case $\beta_{1}^{*}=\beta_{2}^{*}$ ). But if function $f_{\beta}$ is not concave (and in particular it is convex for small $\pi>0$ ), then this bifurcation is subcritical, which implies that two unstable steady states had to exist for $\beta<\beta_{2}^{*}$. The only way in which they could be created is via tangent bifurcation at some smaller $\beta=\beta_{1}^{*}$. Our numeric analysis for different values of $C$ and $b$ demonstrates that such scenario may happen for values of $\phi_{\pi}$ which are very close to $\phi_{\pi}^{w}$. See illustration in Fig. 9.

The RE steady state regains its stability at $\beta=\beta_{3}^{*}$, when function $f_{\beta}$ is convex for small $\pi>0$. Therefore, at $\beta_{3}^{*}$ a subcritical pitchfork bifurcation takes place and two new unstable steady states are created. But this implies (given that $f_{\beta}$ is increasing and bounded) the existence of two other stable non-RE steady states. These five steady state will be also observed for high $\beta$, as we proved in Lemma 4. Thus, we suspect and never disproved through simulations that $\beta_{3}^{*}=\beta_{4}^{*}$.

Proof of Proposition 3.2. According to Proposition B.1(2b) the RE steady state is always locally stable. It is unique and, therefore, globally stable, when function $f_{\beta}$ is concave, i.e., for 
small $\beta$ (see Lemma 3). On the other hand, when $\beta$ is high enough there are two other locally stable steady states, $\pi^{+}$and $\pi^{-}$, see Lemma 4 . These steady states could be created only via tangent bifurcation. Since we cannot rule out the possibility of a number of subsequent tangent bifurcations (when the non-RE steady states are created and disappeared), we denote as $\beta_{1}^{*}$ the instance of the first tangent bifurcation and as $\beta_{2}^{*}$ the instance of the last tangent bifurcation. However, in our numeric analysis for different values of $C$ and $b$ we never encountered a case in which $\beta_{1}^{*} \neq \beta_{2}^{*}$. See illustration in Fig. 9 .

Proof of Proposition 3.3. Since for the aggressive monetary policy $\phi_{\pi}>\phi_{\pi}^{a}>\phi_{\pi}^{w}$, from Proposition B.1(2b) it follows that the RE steady state is locally stable. In order to prove that it is globally stable for every $\beta$, we will show that it is the unique steady state of the dynamics. Since $f_{\beta}$ is an increasing function, uniqueness will imply global stability.

Assume that $\pi>0$. Since function $f_{\beta}$ is bounded from above by a horizontal asymptote $b / \phi_{\pi}$, i.e., $f_{\beta}(\pi)<b / \phi_{\pi}$ for every $\pi$, no steady state can exist within the interval $\left[b / \phi_{\pi}, \infty\right)$. Let us consider $\pi \in\left(0, b / \phi_{\pi}\right)$ and show that $f_{\beta}(\pi) \in\left(0, b /\left(2 \phi_{\pi}\right)\right]$. Since $f_{\beta}$ is an increasing map, $0=f_{\beta}(0) \leq f_{\beta}(\pi) \leq f_{\beta}\left(b / \phi_{\pi}\right)$. Furthermore, since $\phi_{\pi}>\frac{2 b^{2}}{b^{2}-C}$ and $b^{2}-C>0$, we have that $e^{-\beta\left(C-b^{2}+2 b^{2} / \phi_{\pi}\right)} \geq 1$. Combining these inequalities we derive that

$$
f_{\beta}(\pi) \leq f_{\beta}\left(\frac{b}{\phi_{\pi}}\right)=\frac{b}{\phi_{\pi}} \cdot \frac{1-e^{-4 \beta b^{2} / \phi_{\pi}}}{1+e^{-4 \beta b^{2} / \phi_{\pi}}+e^{-\beta\left(C-b^{2}+2 b^{2} / \phi_{\pi}\right)}} \leq \frac{b}{\phi_{\pi}} \cdot \frac{1-e^{-4 \beta b^{2} / \phi_{\pi}}}{2+e^{-4 \beta b^{2} / \phi_{\pi}}} \leq \frac{b}{2 \phi_{\pi}} .
$$

We now showed that there is no fixed point of map $f_{\beta}$ for $\pi>\frac{b}{2 \phi_{\pi}}$.

Suppose, finally, that $0<\pi \leq \frac{b}{2 \phi_{\pi}}$. Applying restrictions $\phi_{\pi}>\frac{2 b^{2}}{b^{2}-C}$ and $b^{2}-C>0$ we find that the condition on $\pi$ implies that $\pi<\frac{b^{2}-C}{4 b}$, so that $4 b \pi<\left(b^{2}-C\right)$ and $C-b^{2}+2 b \pi<\left(C-b^{2}\right) / 2$. We obtain then the following estimate of dynamics on the interval $\left(0, b /\left(2 \phi_{\pi}\right)\right)$

$$
f_{\beta}(\pi)=\frac{b}{\phi_{\pi}} \cdot \frac{1-e^{-4 \beta b \pi}}{1+e^{-4 \beta b \pi}+e^{-\beta\left(C-b^{2}+2 b \pi\right)}} \leq \frac{b^{2}-C}{2 b} \cdot \frac{1-e^{-4 \beta b \pi}}{1+e^{-4 \beta b \pi}+e^{\beta\left(b^{2}-C\right) / 2}} .
$$

Let the function on the right-hand side be denoted as $g(\pi)$. This function is, obviously, increasing in $\pi$, and, with direct computations we find that its first two derivatives are given by:

$$
g^{\prime}(\pi)=2 \beta\left(b^{2}-C\right) \frac{e^{-4 \beta b \pi}\left(2+e^{\beta\left(b^{2}-C\right) / 2}\right)}{\left(1+e^{-4 \beta b \pi}+e^{\beta\left(b^{2}-C\right) / 2}\right)^{2}},
$$

and

$$
g^{\prime \prime}(\pi)=-8 \beta^{2} b\left(b^{2}-C\right)\left(2+e^{\beta\left(b^{2}-C\right) / 2}\right) e^{-4 \beta b \pi} \frac{1-e^{-4 \beta b \pi}+e^{\beta\left(b^{2}-C\right) / 2}}{\left(1+e^{-4 \beta b \pi}+e^{\beta\left(b^{2}-C\right) / 2}\right)^{3}} .
$$

For positive $\pi$ we have that $e^{-4 \beta b \pi} \leq 1$, and hence, the second derivative is always negative. Also 
notice that

$$
g^{\prime}(0)=2 \beta\left(b^{2}-C\right) \frac{1}{2+e^{\beta\left(b^{2}-C\right) / 2}}=\frac{2 x}{2+e^{x / 2}},
$$

where $x=\left(b^{2}-C\right) \beta$. But the function $2 x /\left(2+e^{x / 2}\right)$ has a maximum in a point satisfying $4 e^{-x / 2}+2-x=0$, which is $2 x^{*}$, where $x^{*}$ was defined in Lemma 2 . The value of this maximum is then (approximately) 0.93, which is less than 1 . Therefore, $g^{\prime}(0)<1$.

Since $g$ is concave function for $\pi>0$, the last condition implies that $g(\pi)<\pi$ for every $\pi>0$. Combining it with estimate in (B.6), we conclude that $f_{\beta}(\pi)<\pi$ for all $0<\pi \leq \frac{b}{2 \phi_{\pi}}$. This proves that there is no positive steady state for dynamics given by $f_{\beta}$. Since the function $f_{\beta}$ is odd it also implies that no negative steady state exists. This completes the proof. 


\section{References}

AdAm, K. (2007): "Experimental Evidence on the Persistence of Output and Inflation," The Economic Journal, 117(520), 603-636.

Arifovic, J., J. Bullard, and O. Kostyshyna (2007): "Social Learning and Monetary Policy Rules," Working Paper Series 2007-007, Federal Reserve Bank of St. Louis.

Assenza, T., And M. Berardi (2009): "Learning in a credit economy," Journal of Economic Dynamics and Control, 33(5), 1159-1169.

Assenza, T., P. Heemeijer, C. Hommes, and D. Massaro (2011): "Individual Expectations and Aggregate Macro Behavior," CeNDEF Working Paper 2011-1, University of Amsterdam.

Benhabib, J., S. Schmitt-Grohé, and M. Uribe (2002): “Avoiding Liquidity Traps," Journal of Political Economy, 110, 535-563.

Berardi, M. (2007): "Heterogeneity and Misspecifications in Learning," Journal of Money, Credit and Banking, 31, 3203-3227.

Branch, W. (2004): "The Theory of Rationally Heterogeneous Expectations: Evidence from Survey Data on Inflation Expectations," The Economic Journal, 114(497), 592-621.

Branch, W., And G. Evans (2006): "Intrinsic Heterogeneity in Expectation Formation," Journal of Economic Theory, 127, 264-295.

Branch, W., And B. McGough (2009): "Monetary Policy in a new Keynesian Model with Heterogeneous Expectations," Journal of Economic Dynamics and Control, 33, 1036-1051.

(2010): "Dynamic Predictors Selection in a New Keynesian Model 
with Heterogeneous Expectations," Journal of Economic Dynamics and Control, 34(8), 1492-1508.

Brazier, A., R. Harrison, M. King, and T. Yates (2008): "The Danger of Inflating Expectations of Macroeconomic Stability: Heuristic Switching in an Overlapping Generations Monetary Model," International Journal of Central Banking, 4(2), 219-254.

Brock, W., And P. De Fontnouvelle (2000): "Expectational diversity in monetary economies," Journal of Economic Dynamics and Control, 24(5-7), 725759.

Brock, W., C. Hommes, and F. Wagener (2005): "Evolutionary Dynamics in Markets with Many Trader Types," Journal of Mathematical Economics, 41, $7-42$.

Brock, W. A., and C. H. Hommes (1997): "A Rational Route to Randomness," Econometrica, 65(5), 1059-1095.

Bullard, J., And K. Mitra (2002): "Learning about monetary policy rules," Journal of Monetary Economics, 49(6), 1105-1129.

Carroll, C. (2003): "Macroeconomic expectations of households and professional forecasters," Quarterly Journal of Economics, 118, 269-298.

Cochrane, J. (2005): "Money as Stock," Journal of Monetary Economics, 52, $501-528$.

Cochrane, J. (2010): "Determinacy and Identification with Taylor Rules," NBER Working Paper 13410.

Curtin, R. (2005): "Inflation Expectations: Theoretical Models and Empirical Tests," mimeo, University of Michigan. 
De Grauwe, P. (2010): "Animal Spirits and Monetary Policy," Economic Theory.

Duffy, D., And P. D. Lunn (2009): "The Misperception of Inflation by Irish Consumers," The Economic and Social Review, 40, 139-163.

Evans, G., And S. Honkapohja (2003): "Expectations and the Stability Problem for Optimal Monetary Policies," Review of Economic Studies, 70, 807-824.

(2006): "Monetary Policy, Expectations and Commitment," The Scandinavian Journal of Economics, 108, 15-38.

Evans, G., S. Honkapohja, And K. Mitra (2003): "Notes on agent's behavioral rules under adaptive learning and recent studies of monetary policy," CDMA Working paper 11-02.

Evans, G. W., AND S. HonkAPOHJA (2001): Learning and Expectations in Macroeconomics. Princeton University Press.

Friedman, M. (1968): "The Role of Monetary Policy," American Economic Review, 58, 1-17.

Hommes, C. (2006): "Heterogeneous Agent Models in Economics and Finance," in Handbook of Computational Economics Vol. 2: Agent-Based Computational Economics, ed. by K. Judd, and L. Tesfatsion. Elsevier/North-Holland, 11091186, Amsterdam (Handbooks in Economics Series).

Hommes, C., J. Sonnemans, J. Tuinstra, and H. van de Velden (2005): "Coordination of Expectations in Asset Pricing Experiments," Review of Financial Studies, 18(3), 955-980.

Hommes, C., And F. Wagener (2010): "Does Eductive Stability Imply Evolutionary Stability?," Journal of Economic Behavior and Organization, 75, 25-39. 
Hommes, C. H. (2011): "The Heterogeneous Expectations Hypothesis: Some Evidence from the Lab," Journal of Economic Dynamics and Control, 35, 1-24.

Honkapohja, S., And K. Mitra (2006): "Stability in Economies with Heterogeneous Agents," Review of Economic Dynamics, 9, 284-309.

Howitt, P. (1992): "Interest-Rate Control and Nonconvergence to Rational Expectations," Journal of Political Economy, 100, 776-800.

Kuznetsov, Y. (1995): Elements of Applied Bifurcation Theory. Springer, New York.

LeBaron, B. (2006): "Agent-Based Computational Finance," in Handbook of Computational Economics Vol. 2: Agent-Based Computational Economics, ed. by K. Judd, and L. Tesfatsion. Elsevier/North-Holland, 1187-1232, Amsterdam (Handbooks in Economics Series).

Lines, M., And F. Westerhoff (2010): "Inflation expectations and macroeconomic dynamics: The case of rational versus extrapolative expectations," Journal of Economic Dynamics and Control, 34, 246-257.

MankiW, N., R. Reis, And J. Wolfers (2003): "Disagreement about inflation expectations," in NBER Macroeconomics Annual, ed. by M. Gertler, and K. Rogoff. MIT Press, 209-270.

Manski, C., And D. McFadden (1981): "Alternative Estimators and Sample Designs for Discrete Choice Analysis," in Structural Analysis of Discrete Data, ed. by C. Manski, and D. McFadden. MIT Press, 2-50.

Milani, F. (2007): "Expectations, Learning and Macroeconomic Persistence," Journal of Monetary Economics, 54, 2065-2082.

Pfajfar, D., And E. Santoro (2010): "Heterogeneity, Learning and Information Stickiness in Inflation Expectations," Journal of Economic Behavior and Organization, 75(3), 426-444. 
Pfajfar, D., And B. ZakelJ (2010): "Inflation Expectations and Monetary Policy Design: Evidence from the Laboratory," 2011-091, Tilburg University, Center for Economic Research.

Preston, B. (2005): "Learning about Monetary Policy Rules when Long-Horizon Forecasts Matter," International Journal of Central Banking, 1(2), 81-126.

Sargent, T. J. (1999): The Conquest of American Inflation. Princeton University Press.

TAYlor, J. (1993): "Discretion versus Policy Rules in Practice," CarnegieRochester Conference Series on Public Policy, 39(0), 195-214.

Tuinstra, J., And F. Wagener (2007): "On learning equilibria," Economic Theory, 30(3), 493-513.

Woodford, M. (2003): Interest and Prices: Foundations of a Theory of Monetary Policy. Princeton University Press. 